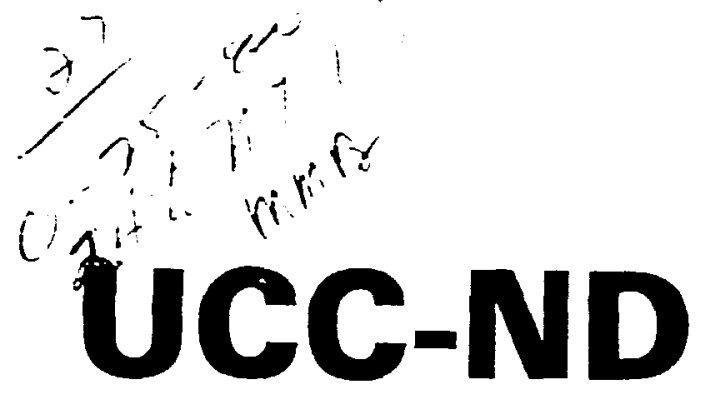

NUCLEAR DIVISION
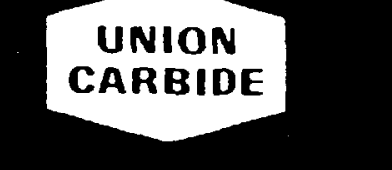

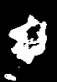

$\checkmark$

is

\section{OPERATEO GY}

UNION CARBIDE CORPOBATION

FOR THE UNITEO STATES

OEPARTMENT OF ENERGY

\section{ETFOD, A Point Model Physics Code with Arbitráry Input}


TABLE OF CONTEATS

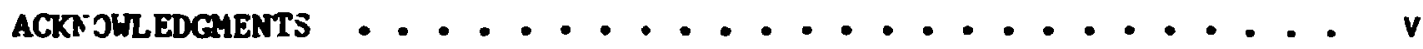
abSTRACT . .......................... l

1. INTROdUCTION ....................... 3

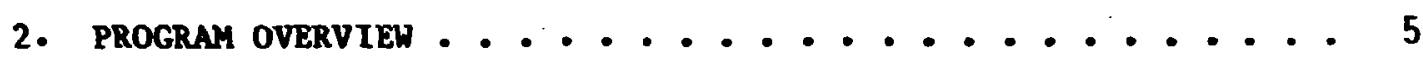

3. DESCRIPTION

3.1 MAIN ..................... 7

3.2 search ...................... 9

3.3 RESID ...................... 11

3.4 FIZICS .................... 12

3.5 PhYSICS PACRAGES ................. 16

3.6 MONIT ........................ 17

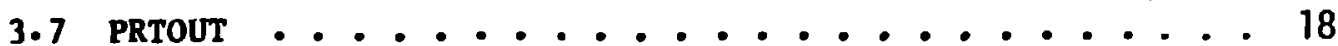

3.8 ETPOD.DAT ................... 19

3.9 INPUT.DAT . . . . . . . . . . . . . . . 21

4. ADDITIONAL HINTS FOR USING ETFOD .............. . 23

4.1 OVERConstrained equations ............... 23

4.2 BADLY UNDERCONSTRAINED RQUATIONS ........... 23

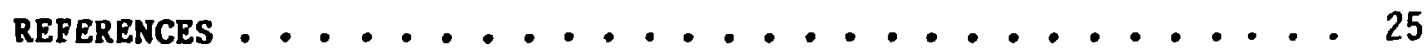
Appendix A

Flowchart .................... 27 Aprendix B

ETFOD.DAT ..................... 29 Appendix C

INPUT.DAT . . . . . . . . . . . . . 3 . Appendix D

Program Listing ................. 33 Appendix E

Sample Cases ................. 47 



\section{ABSTRACT}

ETFOD is a zero-dimensional code which solves a set of physics equations by minimization. The technique used is different than notmally used, in that the input is arbitrary. The user is supplied with a set of Jariables from which he specifies which variables are input (unchanging). The remaining variables become the output. Presently the code 18 being used for ETE reactor design studies. The code was written fin a maner to allow easy modification of -equations, variables, and physics calculations. The solution technique is presented along with hints for using the code. 


\section{INTRODUCTION}

For many years the fusion physics commity has used point model codes to do Tokamak reactor designs. One or more physical equations are written efther in terws of variables defined at a point in the center of the plasma, or else in terus of variables averaged over the plasma volume. Depending on the needs of the users, certain of the jariables are selected as Input and the rest are output variables which define a self-consistent solutior.

Now that reactor design studies are beginning in earnest, the point model codes are being pushed to the limit of their accuracy. The most sophisticated techriques and the most advanced physics models ajallable are being incorporated, whenever fast methods are avallabliz. As a result, it becomes a nontrivial task to use a program to solve any problem other than the one for which it was originally written. When the input variables are changed, elther the code must be extensively rewritten,. or else the code must be used to generate reams of graphic output from which the desired data points may be read by hand.

ETFOD has overcome these problems to very great extent. The user is furnished with a generous list of variables, any subset of which may be identified as input variables. The physics equations may be changed at the whin of the user without fear of destroying the program logic. The secret is to treat the problem as a minimization probiem, so that a self-consiatent solution is found iteratively. Tite solution technique w11l be described in detall in this report. 
2. PROGRAM OVERVIEH

Given a set of equations of the form $y_{1}=F_{1}\left(y_{1}, y_{2}, \ldots, y_{n}\right)$ where the $y_{i}$ are arbitrary variables and the $F_{i}$ are arbitrary functions, one can always write $K_{1}=\frac{y_{1}-F_{1}}{y_{O i}}$ where the $y_{01}$ are the initial values of the variables and tne $R_{1}$ are residuals. The set of equations is then solved by minimizing the residuals. In this fashion ETFOD is designed to solve a set of $0-n$ equations written in residual form. The minimization package used is EO4FAF from the HAG ${ }^{1}$ 1ibrary. EO4FAF minimizes the sum of the squares of the residuals.

The residual equations need initial guesses which are supplied by the user. From the list of eligible variables (see section 3.8) some of the variables may be designated "fixed" while others are free or "active". In general there will be more active variables than there are functions $F_{1}$. In order to fully determine the solution, an aditional set of equations is constructed, also cast in residual form, i.e. $R_{1}=\frac{y_{01}-y_{1}}{y_{01}}$ where $y_{01}$ is the inftial guess for the variable and $y_{1}$ is the current value. Thus the solution obtalned will be that solution of the original equations which is closest to the initial guess.

Since the solution of the physics equations is of primary importance, weights are assigned to the residuals with a relatively large weight given to the physics equations and small weights given to the other equations.

In order to Increase the speed and flextbility of the code, the variables are normalized. This allows unfform restrictions on the sariables regardless of their magnitudes. Each vartable is normalized 
by its intifal guess. Thus the fintial guess given to E04FAF is a vector of $1^{\prime} \mathrm{s}$. When the residuals are to be calculated using the new solution generated by EO4FAF, each element of the solution vector is multiplied by its respective normalization factor, since the physics equations use the actual value of a variable instead of its normalized value.

A Elowchart of the program is listed in Appendix $A$. The nawes of the subroutines wherein the calculations occur are listed to the right of the description. A program listing can be found in Appendix D. Two sample cases are included (Appendix E) to show the effect of changing the weight of an initial guess.

ETFOD runs on the FED PDP-10 and contains calls to machine-dependent $1 / 0$ buffering routines. 


\section{DESCRIPTION}

\subsection{MAIN}

The main piogram initializes the I/O units, opens the data input files and output files, and reads all the input data. The input files are ETFOD.DAT and INPUT.DAT. These files are described in detail in sections 3.8 and 3.9. The output files are ETFOD.OU' and SPECS.ETF. The number of input vartisles from ETFOD.DAT is counted to facilitate changing the vartable 11st. If there are fewer active variables from ETFOD.DAT than physics equations, the problem is overconstrained. A warning is written to unit 5 and ETFOD.OUT. Each active variable in ETFOD.DAT has an associated index number (referring to its relative position in the variable 1ist), weight factor (see section 3.8), and initial value stored in arrays shich are passed in common INDEXP to subroutines FIZICS, RESID, and SEARCH. All the information from file ETFOD.DAT is written to file ETFOD.OUT. The normalized solution vector is infialized to 1.0 . At the end of the run certain plasma parameters are written to ETFOD.OUT. This routine calls SEARCH.

The fol lowing varlables are used in MAIN:

EQNWT weight given to physics residuals.

IND array containing the Indices of the variables read from ETFOD.DAT.

INDP array containing the Indices of the active variables.

IOUT causes the calculation and output of design specifications when set to 1 . (Initially $0_{\text {.) }}$

IX erray containing the weighting flag letters ' $P$ ' (fixed), ' $P$ ' (preferred), and ' $U$ ' (unknown); used in assigning numerical weights to inteial guesses. 
$\mathbf{N}$

number of ac. Ive variables, counted as they are read fron ETFOD.DAT. If MEQ > N, the problem ts overconstrained.

NAMVAR array holding five-letter names of variables, read from ETFOD.DAT.

NIN unit used when reading ETFOD.DAT, defined 6.

NIN2 unit used when reading INPUT.DAT, defined 28.

ivouT untt used when outputting messages to user, defined 5 (defaults to terminal).

NOUT2 unit used when outputting to ETPOD.OUT, defined 20.

NOUT3 unt used when outputting to SPECS.ETP, defined 27.

NVAR total number of variables from ETPOD.DAT.

UNITS array holding 20 ietter unit descriptions of variables in ETFOD.DAT.

WW

array holding the weight factors for the active variables.

WHX

array holding the veight factors corresponding to ' $F$ ' (f ixed), ' $P$ ' (preferred), 'U' (unknown); read from INPUT.DAT. 


\subsection{SEARCH}

Subroutine SEARCH defines the parameters and limits used in E04FAF. All array sizes which are calculated are tested to ensure sufficient space has been allocated. If not, warnings are printed on unit 5 (terminal by default). The inftial guess of the solution is checked, and warnings are written to unit 5 if the residuals are greater than 0.5. The final solution is also tested for accuracy. A warning is written to unit 5 and also to ETPOD.OUT and SPECS.ETF if the physics equations are not accurate within a specified limit. This subroutine is called by the main program and calls RESID, E04FAF, and PRTOUT.

The following variables are used in SEARCH:

APER percent accuracy desired of physics equations. APER $=100$ * FRAACC.

F the sum of the squares of the residuals; returned by E04FAF.

FRAACC the fractional accuracy desired for the physics equations.

FTOL the tolerance criterion used by EO4FAF on $F$. Currently FTOL is initialized to $1.0 \mathrm{E}-6$. On return $\mathrm{FTO}=0.0$ if the value of $B<$ FTOL. FTOL $=1.0$ if a relative test on the range of $F$ 1s satisfied. FTOL $=-1.0$ if the XTOL tolerance test is sarisfied (see XTOL, below). FTOL and XTOL were chosen such that FTOL $=-1.0$ would usually be the tolerance criterion satisfled. This insures that the solution is near a minimum.

IFAIL on return from E04FAF designates the reason for exit from EO4FAF. IFAIL = 1 signals incorrect Input parameters. IFAIL - 2 Indicates that the maximum number of Iterations has been reached but no tolerance conditions are satisfied.

IPRINT specifies the frequency of calls to MONIT, a printout routine. IPRINT -1 causes MONIT to be called every 1teration. IPRINT - 2 causes MONIT to be called only when $P$ 18 reduced. If IPRINT $\leq 0$ MONIT 18 not called. IRC, IW, dimensions of RC, W, X, STEPMX, respectively (see below).

LTEST minimum allowable size of array $W$ required by E04BAF. LTEST $-2 \star M+4 \star N+M \star N+N \star(N+1) / 2+(N+3+N / 3) *(M+2+2 \star N)$. 
MAXCAL maximum number of iterations attempted before E04FAF is forced to exit.

$\mathbf{R C}$

array containing the residuals.

STEPAX array containing restraint values. In a given iteration no active variable can change an amount greater than its associated restraint value in STEPHX. This prevents sudden, large fluctuations of variables which could lead to solutions far from the initial estimates. Every element inftialized to 0.5 .

W worikspace naeded by EO4FAF.

$X$ array containing the current normalized solution. Every element is initialized to 1.0 .

XTOL relative accuracy to which the position of the minimum is required. For two successive iterations whose solution vectors are $\underline{Y}$ and $Z$, the XTOL criterion 1 s satisfied when $\mid Y_{i}$ $-z_{1} \mid<\operatorname{XTOL}^{-} \operatorname{STEPMX}_{1}, 1=1,2, \ldots ., N$ (see FTOL above). 


\subsection{RESID}

Subroutine RESID takes the normalized solution generated by E04FAF and multiplies by the noralizing factors (the laitial guesses). This yields the actual values of the eligible variables which are needed when calculating the physics residuals. The variables ust appear in the data file ETFOD.DAT in the aame order as they appear in commons VARBLS (containing the current values of the variables), INITL (containing the normalfing factors), and INDEX (containing the index numbers which locate values in the other commons). RESID is called by SEARCH and the E04FAF package, and calls FIZICS.

The following variables are used in RESID:

M the total number of residuals.

$\mathbf{N}$ the number of active variables.

$\mathrm{XC}$ array containing E04PAF's current solution (normalized).

$\mathbf{x X}$ arra; containing current solution of al: eligible variables, both active and fixed (normalized; hence those not changing are 1.0 ). 


\subsection{FIZICS}

SUBRUUTINE FIZICS contains the physics equations of the O-D model. The input is primarily from common block VARBLS, which contains the natural values (not normalized) of the elfgible variables. All values are in MRS units unless otherwise noted. FIZICS is called by RESID, and calls the physics packages ALFETA, COULA, INJETA, OHMIC, RADIAT, RATE, RFETA, and TAUS.

FIZICS compites all the residuals, both for the physics equations and the initial value equations. (See the description ia section 2.) The physics equations are subject to constant revision and improvement, but the following set of equations is representative. (Refer to the table of variableg for identification of symbols.)

$$
\begin{aligned}
& R_{1}=\frac{1}{N_{10}}\left[N_{1}-N_{e}(1-z \xi)\right] \\
& R_{2}=\frac{1}{N_{10}}\left[N_{1}-N_{e}\left(z_{\text {eff }}-z^{2} \xi\right)\right]
\end{aligned}
$$

where $\xi=N_{2} / N_{e}$

$$
\begin{aligned}
& R_{3}=\frac{1}{R_{0}}\left[\beta-\frac{\left(N_{1} T_{1}+N_{e} T_{e}+N_{2} T_{1}\right) 1.602 \times 10^{-16}}{2 \mu_{0} B_{t}^{2}}\right] \\
& R_{4}=\frac{1}{P_{\alpha 0}}\left[P_{\alpha}-\frac{3}{2} \frac{\left(N_{1} T_{1}+N_{e} T_{e}+N_{2} T_{1}\right)}{\tau_{E}} 1.602 \times 10^{-16}\right]
\end{aligned}
$$




$$
R_{5}=\frac{1}{P_{\alpha 0}}\left[P_{\alpha}-\frac{N_{i}^{2}<0 v>Q_{\alpha}}{4}\right]
$$

where $Q_{a}=5.607 \times 10^{-13}$ joule $(3.52 \mathrm{MeV})$

$$
\begin{aligned}
& R_{6}=\frac{1}{P_{a 0}}\left[P_{a} n_{a}+P_{i n j} n_{i n j}+P_{r f^{n}} r f+F_{c o u l}\right. \\
& \left.-\frac{3}{2} \frac{T_{1}\left(1.602 \times 10^{-16}\right)}{C_{1}}\left(\frac{1}{C_{T P^{\top} T P}}+\frac{1}{C_{N C^{\top} \mathrm{NC}}}+\frac{1}{C_{R T^{\top} R T}}\right)\right] \\
& \left.R_{7}=\frac{1}{P_{\alpha u}}\left[p_{\alpha(1}-n_{\alpha}\right)+p_{i n j}\left(1-n_{\text {inj }}\right)+p_{\left(1-n_{r f}\right.}\right)-P_{\text {coul }} \\
& \left.-P_{\text {rad }}+P_{\Omega}-\frac{3}{2} \frac{T_{e}\left(1.602 \times 10^{-16}\right)}{C_{e}} \frac{1}{C_{\text {EMP }}{ }_{\text {EMP }}}\right] \\
& R_{8}=\frac{1}{P_{f_{0}}}\left[P_{f}-5_{P_{Q}} V\right] \\
& R_{9}=\frac{1}{w_{\alpha 0}}\left[w_{\alpha}-\frac{4}{5}-\frac{P_{f}}{5}\right] \\
& R_{10}=\frac{1}{I_{p o}}\left[I_{p}-\frac{5.6 \times 10^{6} a B_{t}\left(1+\sigma^{2}\right) 1.11}{28 A\left(1-\frac{1}{A^{2}}\right)^{2}}\right]
\end{aligned}
$$


The following variables are used in FIzICS:

(Symbol a) Minor radius of the plassa (a).

AlEXT External inductance $(H)$.

ALPI Internal inductance (H).

AR (Symbol A) Aspect ratio.

AREA (Symbol S) Surface area of first wall $\left(m^{2}\right)$.

BP Pololdal magnetic field (T).

BT Toroidai magnetic field (T).

C.C (Symbol R) The array of residual values. The first MEQ values are the physics residuals. The last M-MEQ values are the initial valur. residuals.

CE (Symbol $C_{e}$ ) Hultiplier for electron conduction and convection confinement time.

CEMP (Symbol C EMP) Multiplier for empirical confinement time.

CI (Symbol $C_{f}$ ) Multiplier for Ion conduction and convection confinement time.

CNC (Symbol $C_{N C}$ ) Multiplier for neoclassical confinement $t$ ime.

CRT (Symbol $C_{R T}$ ) Multiplier for Ripple Trapping confinement time.

CTP (Symbol $C_{T P}$ ) Hultiplier for Trapped Particle confinement time.

EB Neutral beam energy (primary component, in keV).

EQNWT A weighting factor used to give the physics residuals more weight than the initial value residuals.

ETALP (Symbol $n_{a}$ ) The fraction of alpha power absorbed by the lons.

ETAINJ (Symbol $n_{\text {inj }}$ ) The fraction of injected powe: absorbed by the 1ons.

ETARP (Symbol $n_{r f}$ ) The fraction of $r f$ power absorbed by the lons.

IP (Symbol $I_{p}$ ) Plasma current (A).

NE (Symbol $N_{e}$ ) Electron denetey $\left(m^{-3}\right)$.

NI (Symbol $\mathrm{N}_{1}$ ) Ion density $\left(\mathrm{m}^{-3}\right)$.

N2

(Symbol $N_{2}$ ) Impurity density $\left(m^{-3}\right)$. 


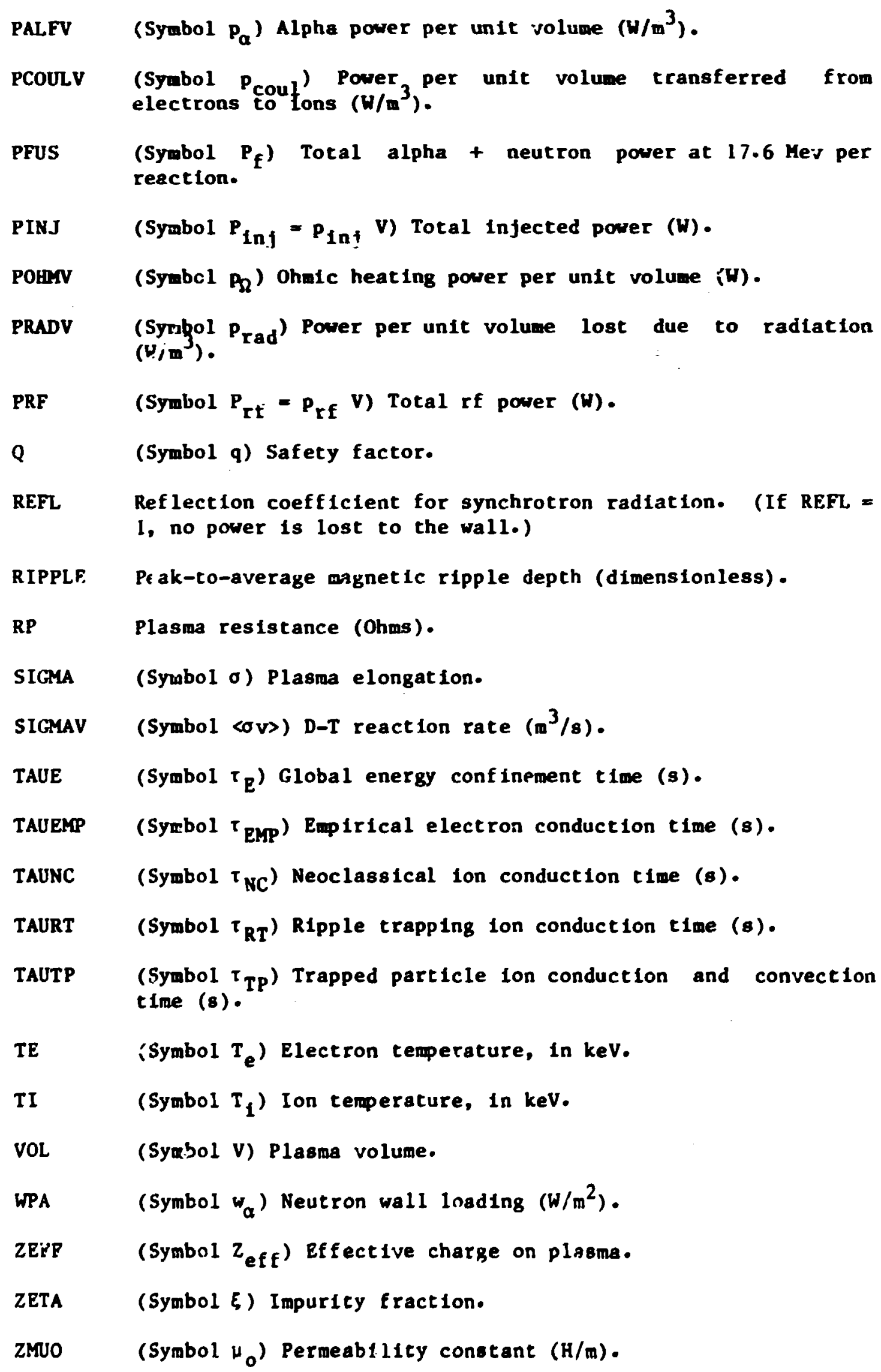




\subsection{Physics Packages}

The physics portion of the ETFOD code is highly modularized to peralt upgrading of the physics models as new models become available. Where possible, the physics packages are borrowed from or benchmarked against those of the WHIST $1-D$ transport code. 2

The following subroutines are among the current physics packages:

ALFETA Computes the fraction of the alpha power which is absorbed by the Ions.

CounM Computes the electron-1on power transfer due to Coulnmb colliston.

INJETA Computes the traction of neutral beam energy ajsorbed by the lons.

OHMIC Computes the Ohmic heating rate.

RADIAT Computes the power loss due to radiation.

RATE Computes the D-T fusion reaction rate.

RFETA Computes the fraction of $x$ power absorbed by the lons.

TAUS Computes confinement times predicted by theoretical and empirical models.

The above routines are very primftive at the present time. They will be described in detail in a later memo, after the code has been upgraded to include shape factors and more sophisticated physics models. 


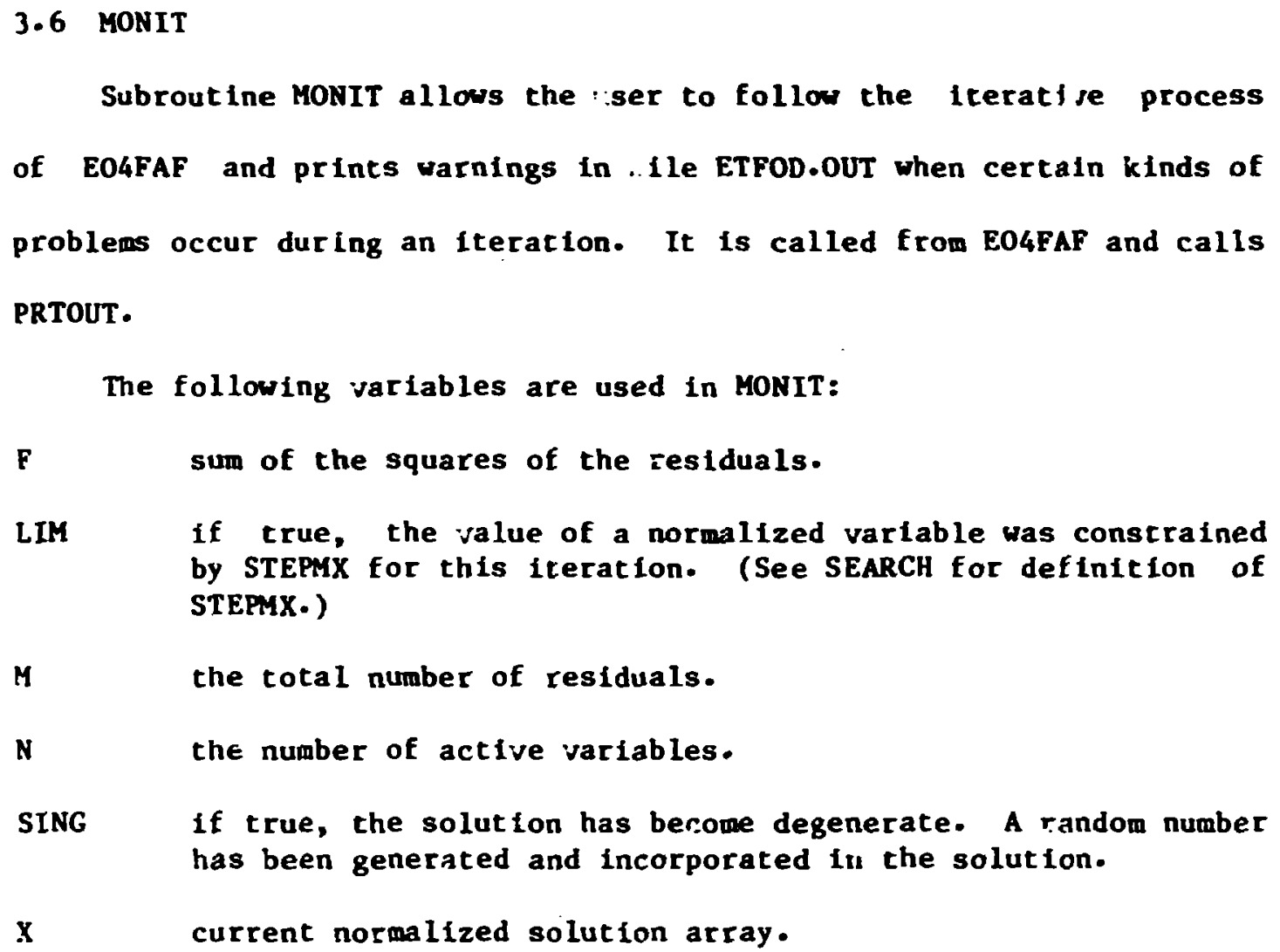




\subsection{PRTOUT}

Subroutine PRTOUT wites out the variable names and their current salues. The values of the fixed variakles are followed by an ' $F$ '. PRTOUT is called from SEARCH and MONIT. 


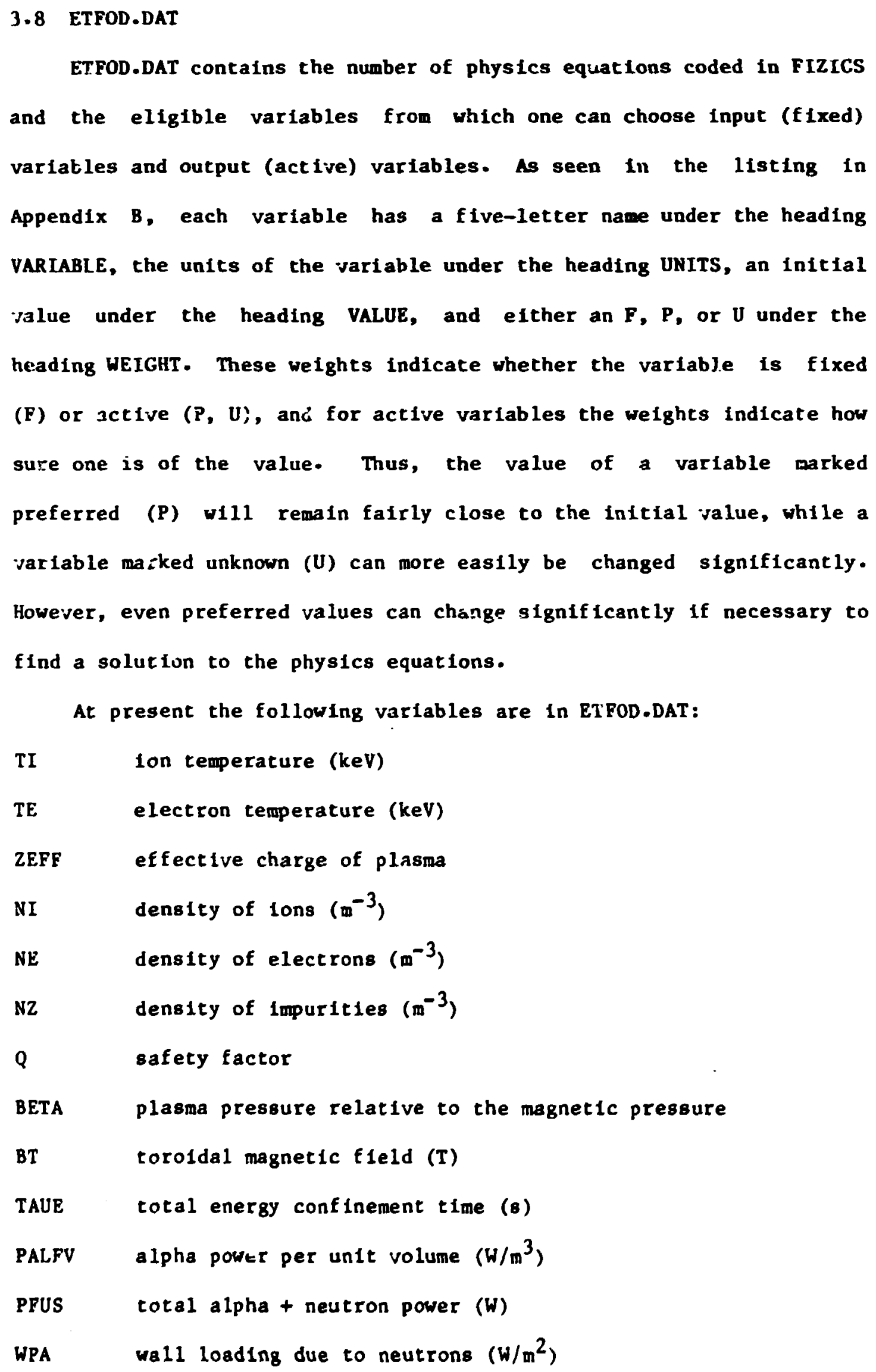




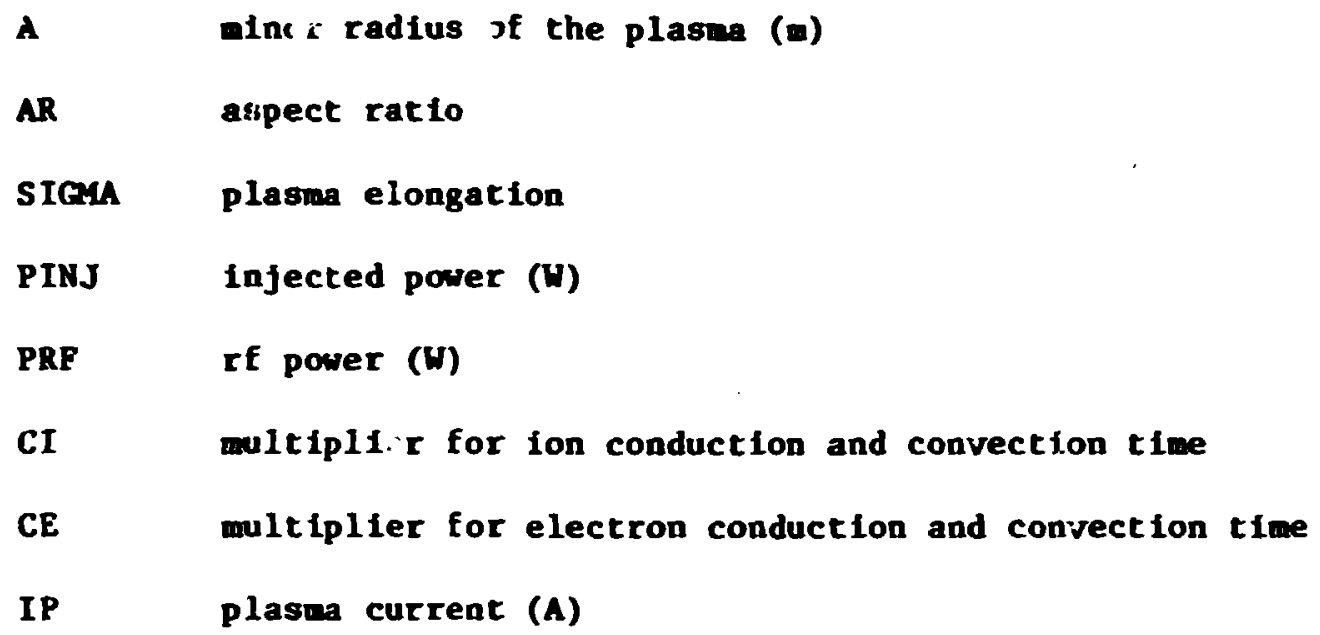




\subsection{INPUT.DAT}

The data in INPUT.DAT is read in from MAIN and stored in common INP. See Appendix C for a listing of the file. The values remain unchanged.

The following variables are in INPUT.DAT:

WWX(1) the weight given to fixed variables in ETFOD.DAT

WWX(2; the weight given to preferred variables in ETFOD.DAT

WWX(3) the weight given to unknown variables in ETFOD.DAT

EQWWT the weight given to the physics residuals in FizICS

$2 \quad$ charge of impurity ion

CTP multiplier for trapped particle confinement time

CNC multiplier for neoclassical confinement time

CRT multiplier for ripple trapping confinement time

CEMP multiplier for empirical confinement time

AMU mass of plasma ions (AMU)

AMZ mass of impurity fons (AMU)

EB neutral beam energy (prinary component, in keV)

LI Internal inductance parameter (dimensionless)

REFL reflection coefficient for synchrotron radiation

RIPPLE peak-to-average magnetic ripple depth (dimensionless)

FACTOR low beta multiplier for plasma current

GAMAA registive loss factor

SCAPL scrape of $f$ layer width (m)

PBURN burn pressure (TORR)

PQNCH quench pressure (TORR) 
4. ADDITIONAL HINTS FOR USING ETFOD

Occasionally the code w11 find a solution to the least squares problem which is not a solution to the physics equations. (If so, there is an error message in the output file.) When this occurs, we have found it to be due to one of two things: either the equations are overconstrained or else they are badly underconstrained.

\subsection{Overconstrained Equations}

If there are fewer free variables than physics equations, the program prints an error message but solves the problen anvway. If the initial guess is very good, the solution to the physics equations may still be found. It is also possible that a certain subset of the physics equations is overconstrained. The program cannot tell you whether this has happened, but you shouid suspect that it has if all the output values seem very reasonable, but nevertheless the physics equations do not quite balance. The remedy is to float another variable or two. If this fatls to work, then one or more of your initial guesses is much too low.

\subsection{Badly Underconstrained Equations}

This problem tends to occur when developing a brand new data set. The initial guesses are frequently poor, and there are usually a large number of free variables (free - "preferred" or "unknown"). The solution will contain values that are way out of line with the values expected. Also the execution time may be very long, especially if the Inftial guesses are too low (it to better to guess large values if unsure). The remedy is :0 tighten constraints on wayward variables. 
REFERENCaS

1. Numerical Algorithms Group Subroutine Library, NAG US Inc., Downers Grove, Illinois 60515 .

2. A. T. Mense, W. A. Houlberg, S. E. Attenberger, "A 1-D Transport Model for Analysis of Expanding Radius and Full Bore Startup of Tokamak Plasmas," ORNL/TM-6841. 
ONme.tos 20.2197 FE

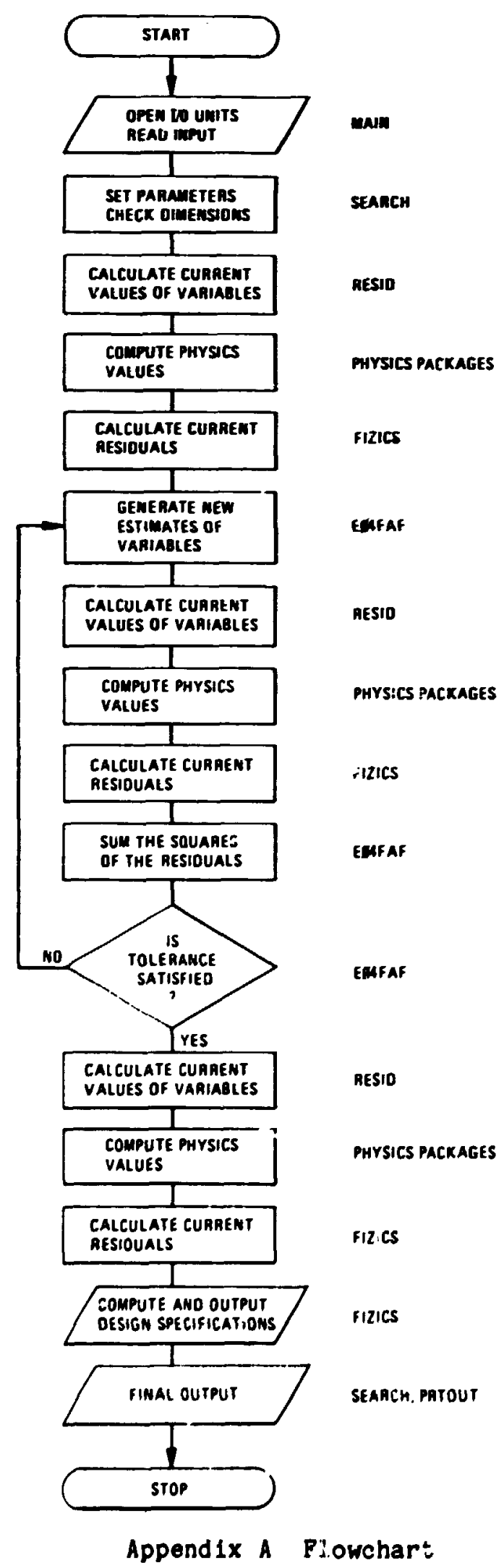


Appendix B ETFøD.DAT

\begin{tabular}{|c|c|c|c|c|c|}
\hline TEST & $\begin{array}{l}\text { INPUT DATA. } \\
10=\text { NUMBEF }\end{array}$ & $\begin{array}{l}\text { TNS RI } \\
\text { OF } \mathrm{col}\end{array}$ & $\begin{array}{l}\text { F. DESIGN } \\
\text { STRAINT E }\end{array}$ & $\begin{array}{l}\text { OALL/TM-6721 } \\
\text { 2UATIONS }\end{array}$ & $\begin{array}{l}F=F \text { IXED } \\
\text { P=PREFERRED VALUE }\end{array}$ \\
\hline WVAR & VALUE & MEIGHT & VARIABLE & UNiTS & U=UNRNOWN \\
\hline 1 & $12.00 E 0$ & $\mathbf{F}$ & $\because I$ & (REV) & \\
\hline 2 & $12.00 \mathrm{EO}$ & $\mathbf{F}$ & TE & (KEV) & \\
\hline 3 & 1.500 & $\mathbf{P}$ & ZEFE & & \\
\hline 4 & 1.70 E2O & $P$ & MI & $(M-1)-3)$ & \\
\hline 5 & $1.70 E 20$ & $\mathbf{P}$ & WE & $(\mu-3)$ & \\
\hline 6 & $0.54 \mathrm{E} 19$ & $\mathbf{P}$ & NZ & $\left(y^{n}=3\right)$ & \\
\hline $\begin{array}{l}7 \\
8\end{array}$ & $\begin{array}{r}3.8 \\
7 . E-2\end{array}$ & $\begin{array}{l}F \\
F\end{array}$ & $\begin{array}{l}0 \\
\text { BETA }\end{array}$ & (SAFETY FACTOR) & \\
\hline 9 & $5.01 \mathrm{EO}$ & 5 & BT & (TESLA) & \\
\hline 10 & 1.500 & U & TAUE & $(\mathrm{SEC})$ & \\
\hline 11 & $0.920 E 7$ & U & PALFV & $\left(W / M^{2}=3\right)$ & \\
\hline 12 & $1.042 \mathrm{E} 10$ & U & PFUS & (w) & \\
\hline 13 & $3.300 E 7$ & $\mathbf{P}$ & WPA & $(H / M=2)$ & \\
\hline 14 & 1.34 & $\mathbf{F}$ & A & (H) & \\
\hline 15 & 4.179 & $\mathbf{F}$ & AR & & \\
\hline 16 & 1.6 & $\mathbf{F}$ & SIGMA & & \\
\hline 17 & $0 . E-00$ & $\mathbf{F}$ & PINJ & (H) & \\
\hline 18 & 1.E-24 & $\mathbf{P}$ & PRF & (W) & \\
\hline 19 & $1.0 r$ & $\mathbf{P}$ & CI & & \\
\hline 20 & 1.00 & $\mathbf{P}$ & $C E$ & & \\
\hline 21 & 5.0E6 & U & IP & (AMPS) & \\
\hline END & & & & & \\
\hline
\end{tabular}




\section{Appendix C INPUT.DAT}

$\begin{array}{rrrrr}\text { WWX(1) } & \text { WWX(2) } & \text { WWX(3) } & \text { EQNHT } & \\ 1.000 & 0.040 & 0.010 & 10.0 & \\ 2 & \text { CTP } & \text { CNC } & \text { CRT } & \text { CEMP } \\ 8.0 & 1 \text { OE20 } & 1.0 & 6.0 E 03 & 1.0 \\ \text { AMU } & \text { AMZ } & \text { EB } & \text { LI } & \text { REFL } \\ 2.5 & 42 . & 150.0 & 1.50 & 0.9 \\ \text { RIPPLE } & \text { FACTOR } & \text { GAMMA } & \text { SCRAPL } & \text { PBURN } \\ \text {.0155 } & 0.8 & 0.1 & 0.250 & 0.1 E-5 \\ \text { FQNCH } & & & & \\ 0.10 E-3 & & & & \end{array}$


Appendix D Program L:sting

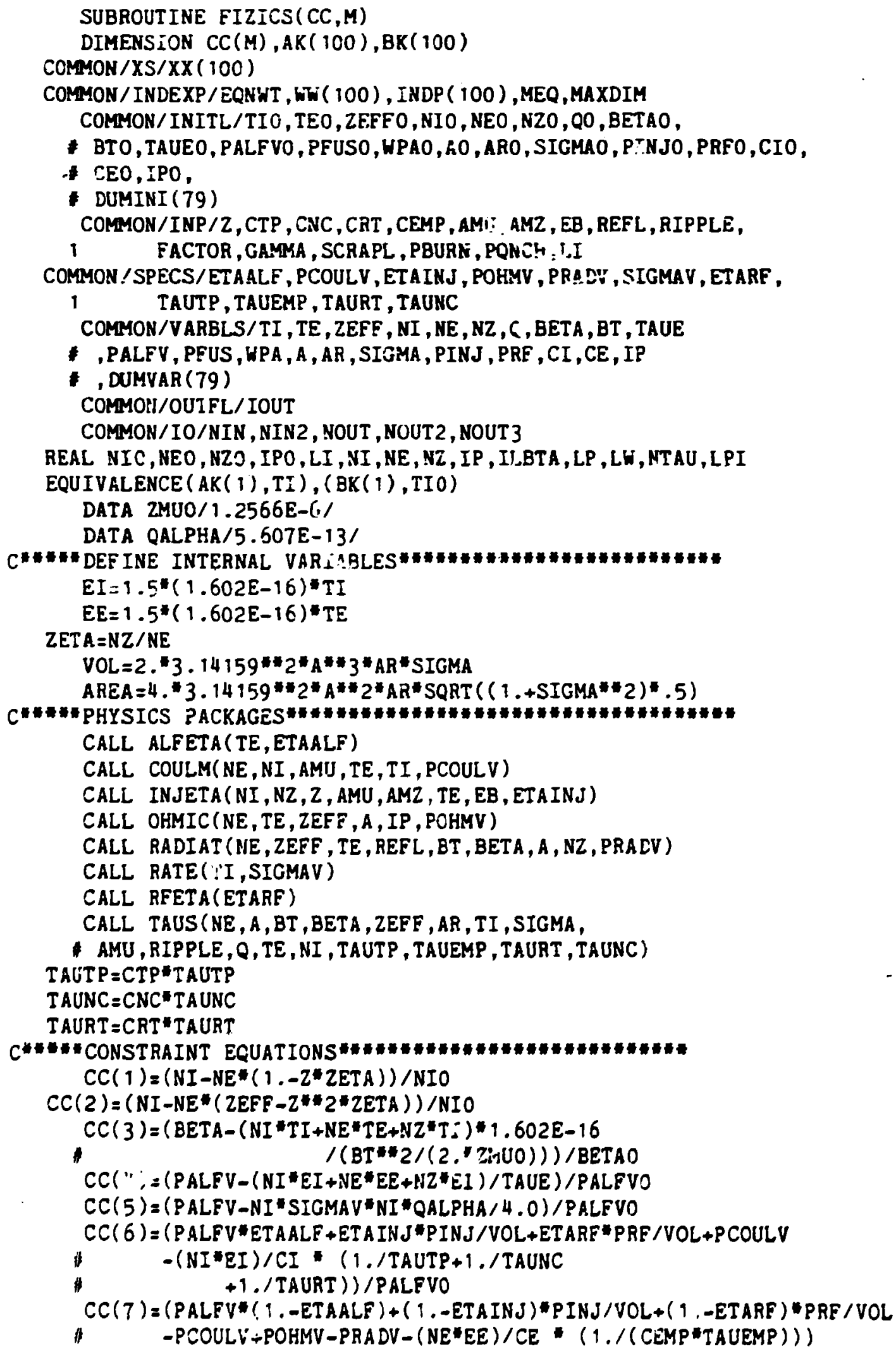




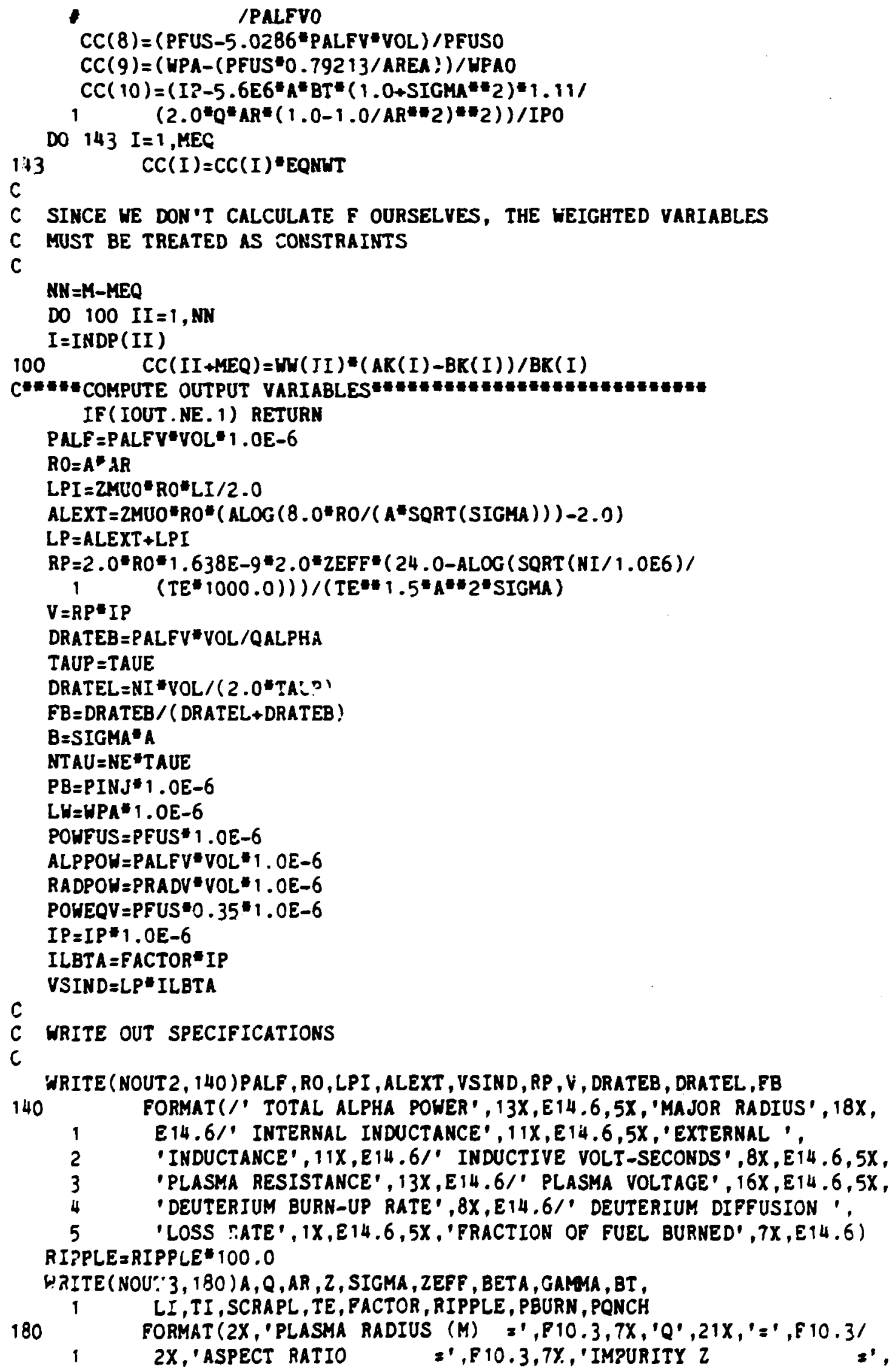




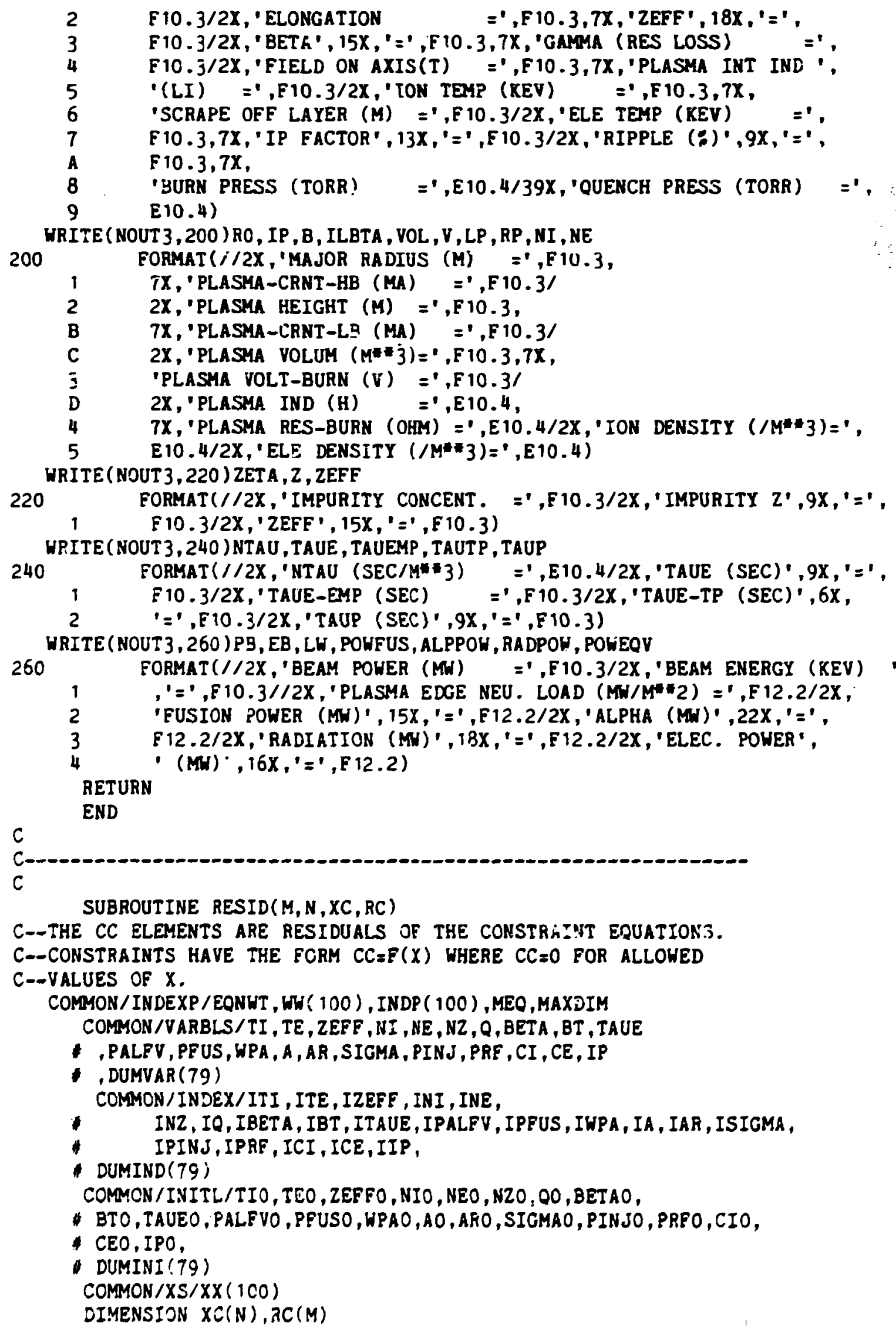


REAL NIO, NEO, NZO, IPO , LI , NI, NE, NZ, IP , ILBTA , LP , LH, NTAU, LPI C--VARIABLES MUST APPEAR IN THE DATA FILE IN THE SAME ORDER C--AS THEY APPEAR IN COMMON.

C

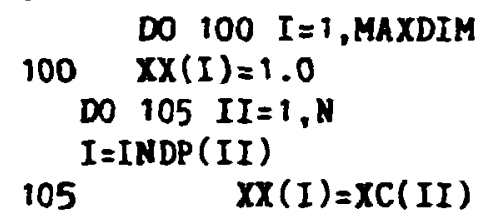




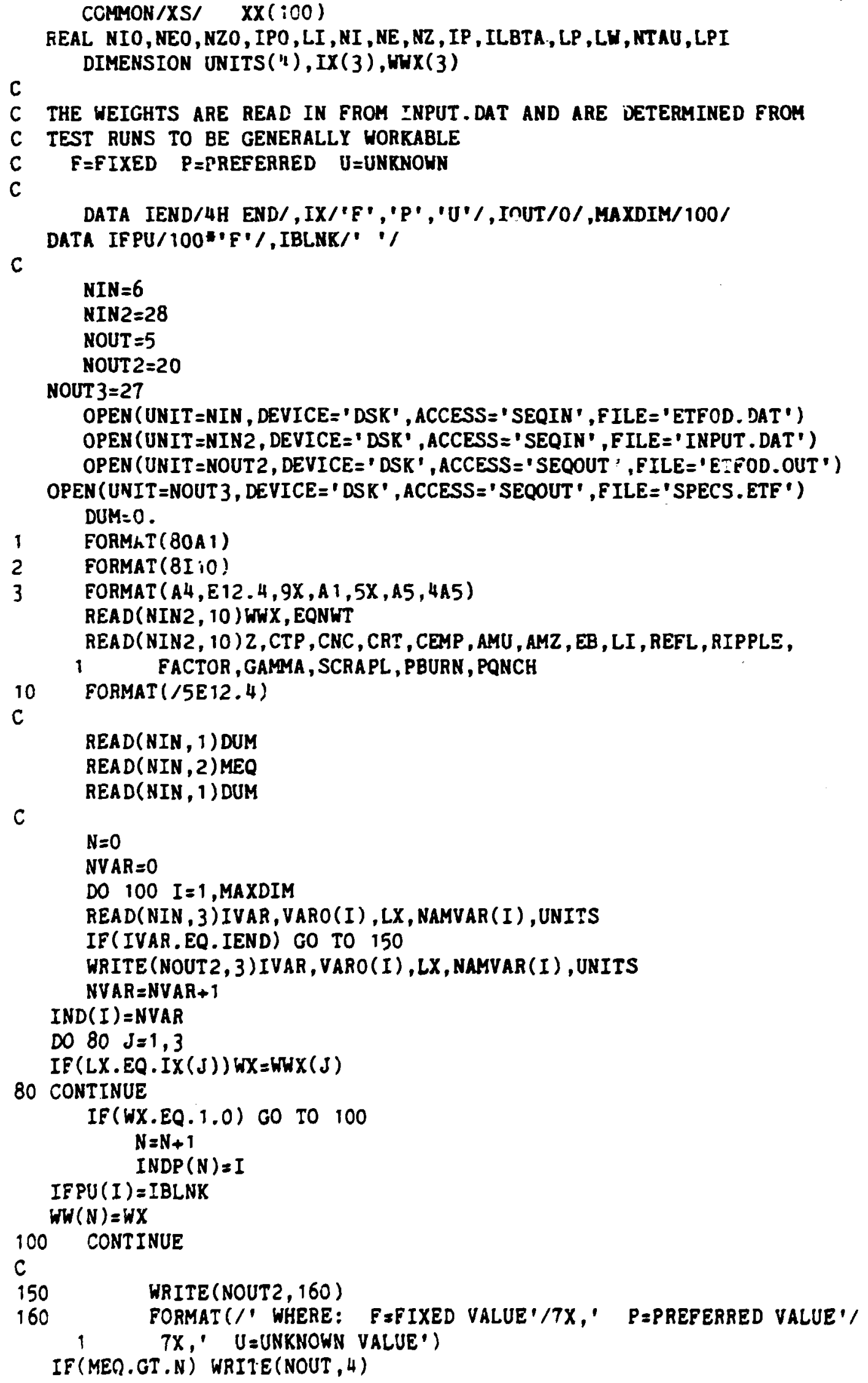




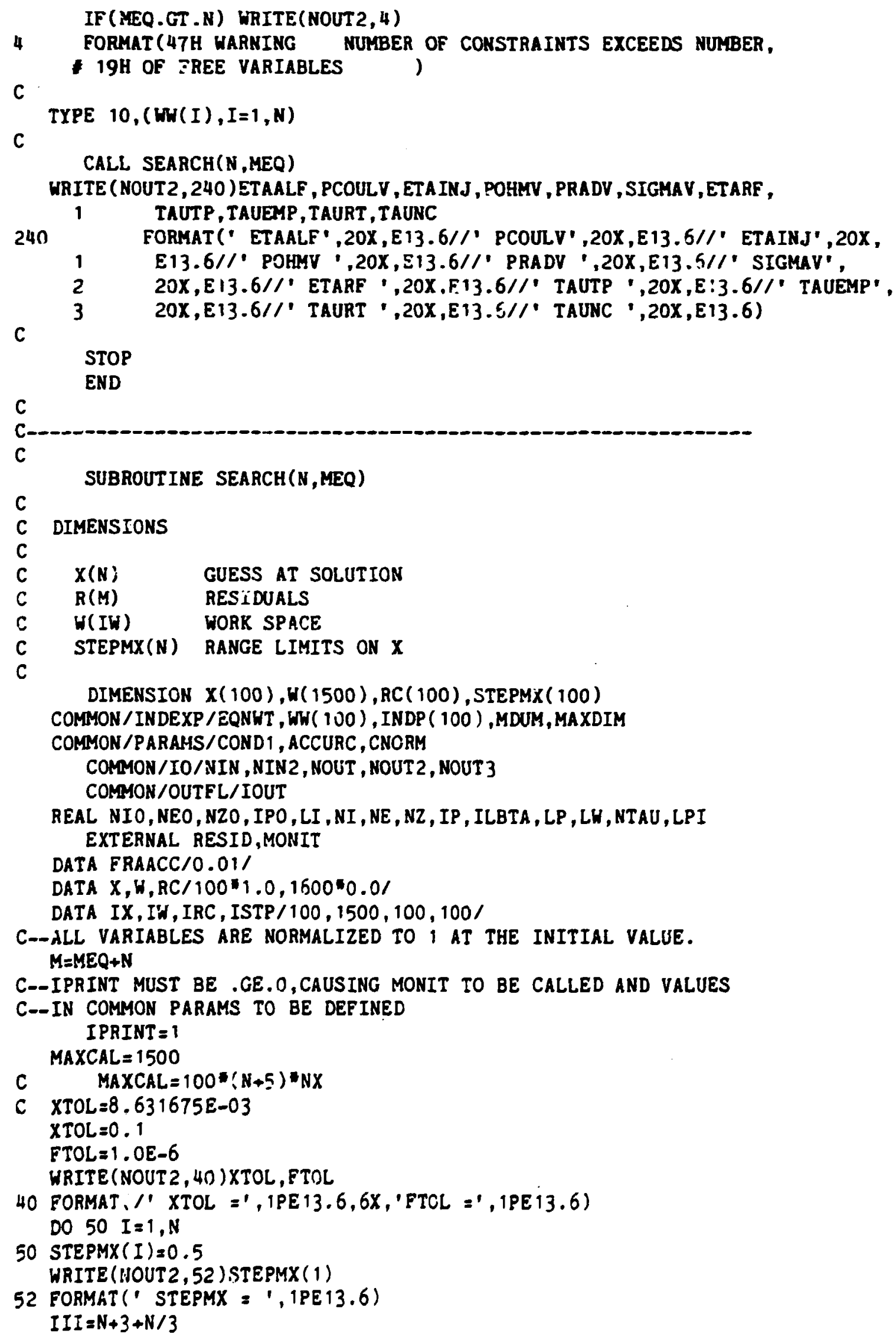




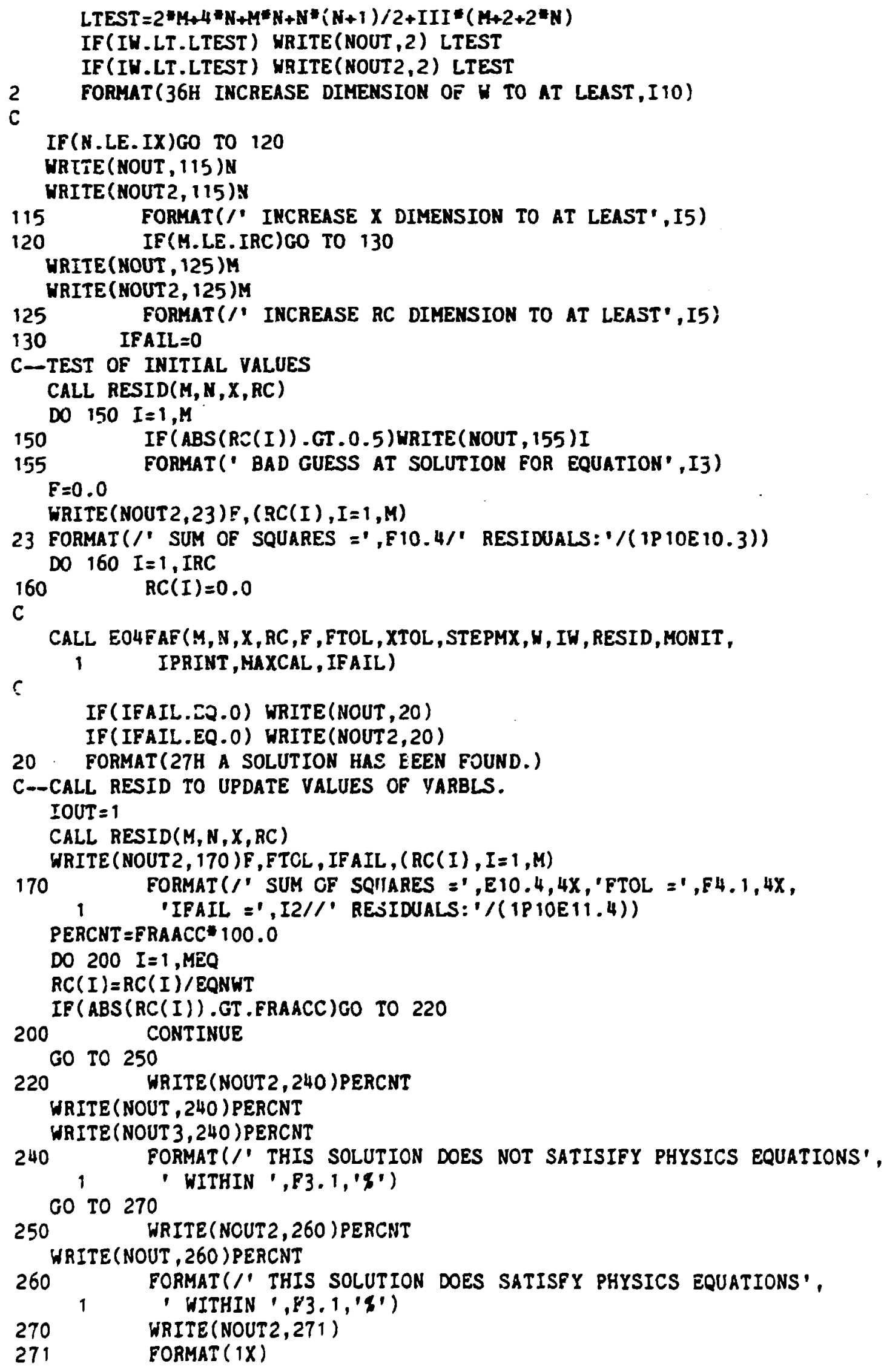




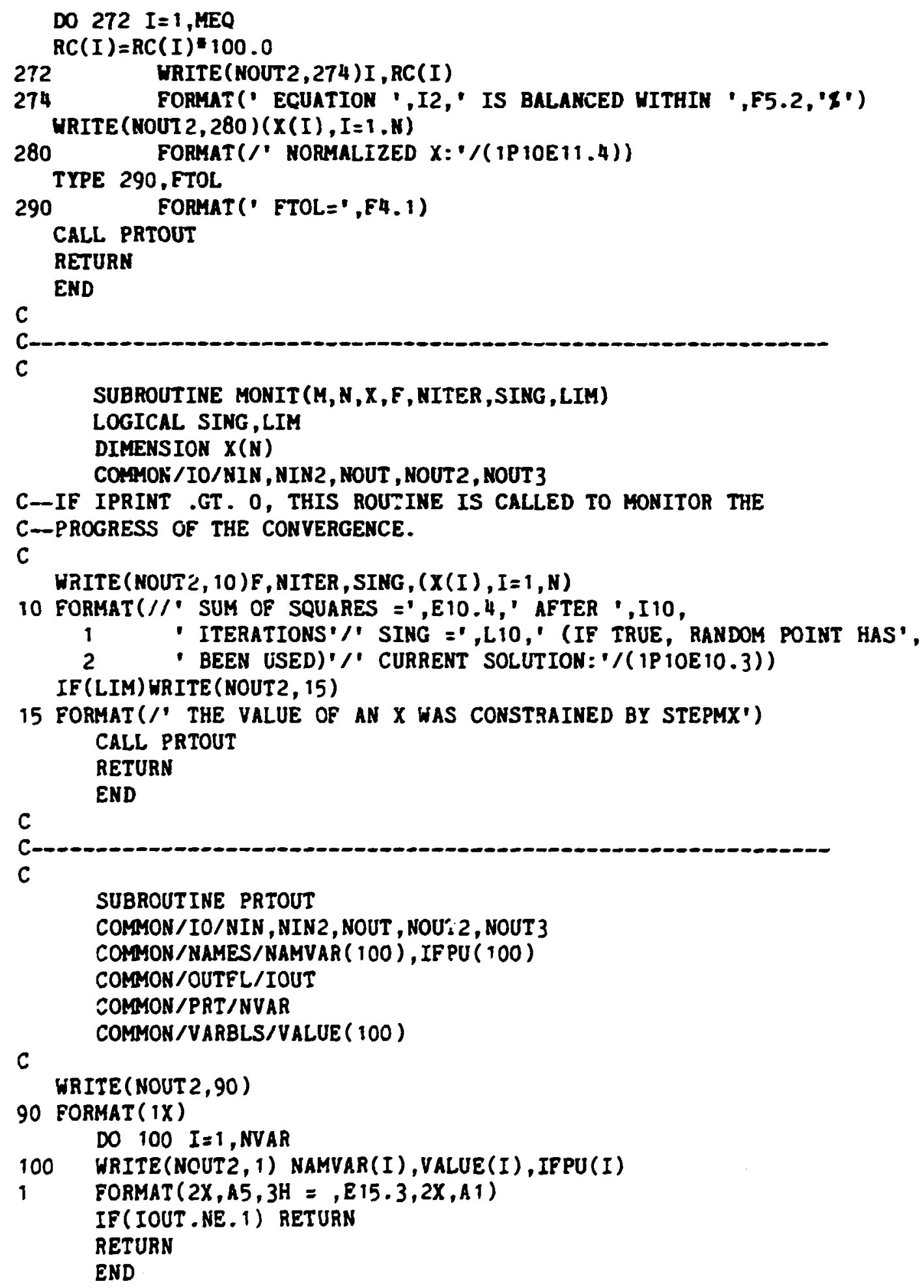




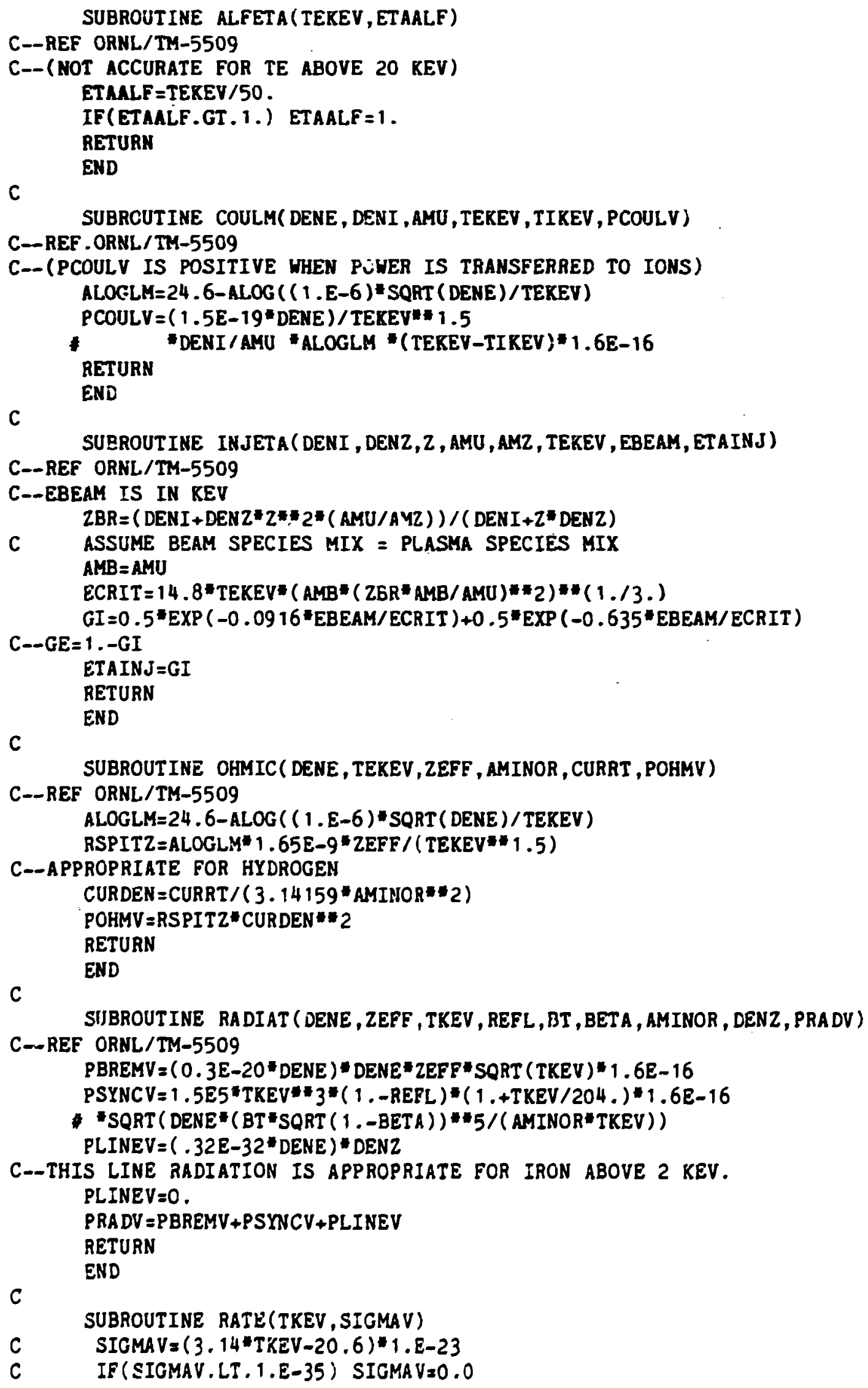




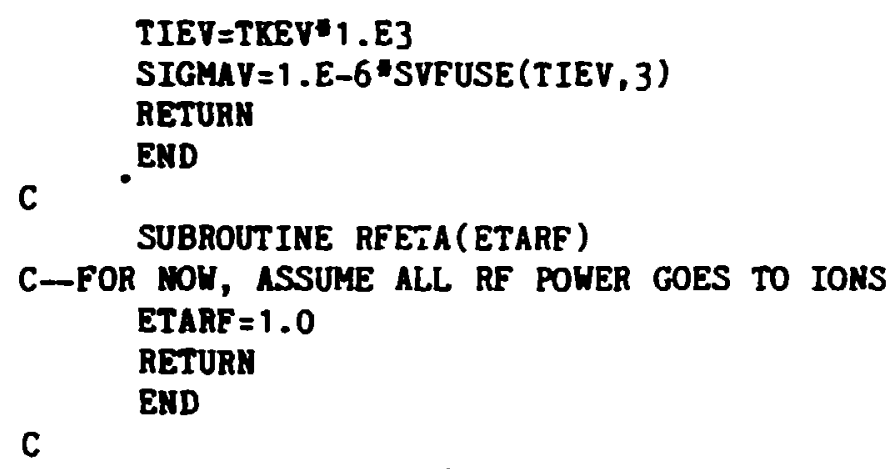

SUBROUT INE TAUS (DENE, AMINOR, ET, BETA, ZEFF, ASP, TIKEV, ELONG,

- AMU, DELTA, QS, TEKEV, DENT, TAUTP, TAUEMP, TAURT, TAUKC)

C-TRAPPED PARTICLE MODE FROM MARTIN PENG

TAUTP $=(2.4 E 20 / D E N E)$

- (AMINOR/1.2)*4.

- $\quad(B T / 5.3) * 6$

- (BETA/0.07)=2

- (2EFF/1.1)

- (ASP/4.17) 2.5

- (12./IIKEV $) \div 5.5$

- $\quad(i .+$ ELONG*:2)/(2.*i.78)

C--EMPIRICAL SCALING FROM MARTIN PENG

TAUEMP $=(5 . E-21$ DENE $)$ AMINOR * 2

C-RIPPLE TRAPPING DERIVED FROM STRIMGER'S DIFF. COEFF.

RO $=A S P$ * AMINOR

ZNUE $=1.6 E 4^{*}$ ZEFF" (DENE/1.E 19)*(TIREV)*-1.5

SQRAM $=$ SORT $\left(A M U * 18 ; \dot{N}^{-}\right)$

BTKG $=B T=10$.

$V D=1 . E 6 * \mathrm{TIKEV} /(\mathrm{BTKG} * \mathrm{RO})$

$X R T=46.5 * D E L T A * 1.5 *$ SQRAM*VD**2/(ZNUE*ELONG*2)

TAURT $=$ AMINOR * $2 /(4 . * X R T * 1 . E-4)$

C-NEOCLASSICAL DIFFUSION DERIVED FROM DIAGONAL TERMS OF

C--HINTON-MOORE MODEL FOR ION THERMAL CONDUCTIVITY.

$B P=B T *((1,+E L O N G * 2) / 2) /.(Q S * A S P)$

EPS $=1.1$ ASP

$Z L=24$. $-A L O G((1, E-6) *$ SORT (DENE)/TEKEV)

ZNUI $=6.71 E-14 * D E N I * Z L /($ TIKEV*1.E3)*1.5

OMEGBI $=9.78 E 3 * B P /\left(B *^{* R O}\right) * S Q R T(1 . E 3 * T I K E V /(E P S * A M U))$

SNI $=2$ NUI $/($ EP.'OMEGBI)

RHOIT: $14 \%$. 25E-04"SQRT (AMU*1.E3"TIKEV)/BP

$X X 2=.66 \div(1 . /(1 .+1.03 *$ SQRT (SNI $))+.31 *$ SNI $)$

- +EPS*3*1.77*SNI/(1,+.74*SNI"EPS* 1.5)

XINC=SQRT $(E P S) *$ ZNUT *RHOIT $2 * X K 2$

TAUNC =AMINOR* $2 /(4 . *$ XINC*1.E-4)

RETURN

END

FUNCTION SVFUSE(TI, IHATE)

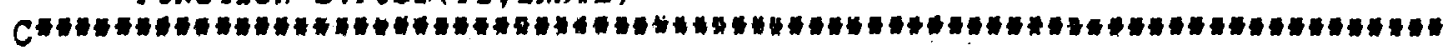

C*E*WW.A.HOULBERG ORNL $2 / 79$.

C* TABULATED DATA FROVIDED BY J.R.MCNALLY, JN.

C

7**:*SVEUSE IS 〈SIGMA VEL> AVERAGED OVER TWO MAXWELLIAN DISTRIBUTTONS

C* WITH TEMPERATURE TI. UNITS ARE IN CM*3/SEC FOR SVPUSE.

CW" IRATE=INDEX DENOTING REACTION 
$9.84 \mathrm{E}-18,1.03 \mathrm{E}-17,1.09 \mathrm{E}-17,1.14 \mathrm{E}-17,1.19 \mathrm{E}-17$, $1.24 \mathrm{E}-17,1.29 \mathrm{E}-17,1.34 \mathrm{E}-17,1.39 \mathrm{E}-17,1.44 \mathrm{E}-17$, $1.49 \mathrm{E}-17,1.54 \mathrm{E}-17,1.59 \mathrm{E}-17,1.64 \mathrm{E}-17,1.69 \mathrm{E}-17$, $1.73 \mathrm{E}-17,1.78 \mathrm{E}-17,1.83 \mathrm{E}-17,1.88 \mathrm{E}-17,1.93 \mathrm{E}-17$, $1.97 \mathrm{E}-17,2.02 \mathrm{E}-17,2.07 \mathrm{E}-17,2.12 \mathrm{E}-17,2.16 \mathrm{E}-17$, 2.21E-17, 2.66E-17, 3.09E-17, 3.51E-17, 3.90E-17, 4.29E-17/

DATA SV3/6.27E-21,1.86E-20,4.44E-20,9.11E-20,1.67E-19, 2.83E-19,4.47E-19,6.72E-19,9.67E-19,1 . 34E-18, $1.81 E-18,2.38 E-18,3.06 E-18,3.86 E-18,4.79 E-18$, $5.86 \mathrm{E}-18,7.07 \mathrm{E}-18,8.43 \mathrm{E}-18,9.95 \mathrm{E}-18,1.16 \mathrm{E}-17$, $1.35 \mathrm{E}-17,1.55 \mathrm{E}-17,1.77 \mathrm{E}-17,2.00 \mathrm{E}-17,2.26 \mathrm{E}-17$, 2.53E-17, 2.81E-17,3.12E-17,3.44E-17,3.78E-17, $4.14 E-17,4.52 E-17,4.91 E-17,5.31 E-17,5.74 E-17$, E.17E-17,6.63E-17,7.09E-17,7.57E-17,8.07E-17, 8.57E-17,9.09E-17,9.62E-17,1.02E-16,1 .07E-16, 1. 13E-16,1 .74E-16,2.39E-16,3.06:-16,3.70E-16, $4.31 E-16,4.88 E-16,5.39 E-16,5.86 \mathrm{e}-16,6.28 E-16$, $6.65 \mathrm{E}-16,6.98 \mathrm{E}-16,7.27 \mathrm{E}-16,7.52 \mathrm{E}-16,7.74 \mathrm{E}-16$, $7.93 E-16,8.09 E-16,8.23 E-16,8.35 E-16,8.45 E-16$, 8.54E-16,8.61E-16,8.66E-16,8.70E-16,8.74E-16, 8.76E-16,8.77E-16,8.78E-16,8.78E-16,8 .77E-16, 8.76E-16,8.7": E-16,8.72E-16,8.70E-16,8.67E-16, $8.64 \mathrm{E}-16,8.61 \mathrm{E}-16,8.57 \mathrm{E}-16,8.54 \mathrm{E}-16,8.50 \mathrm{E}-16$, $8.46 \mathrm{E}-16,8.42 \mathrm{E}-16,8.37 \mathrm{E}-15,8$. 33E-16,8 .29E-16, 8.24E-16,7 .77E-1E,7.31E-16,6 89E-16,6 .50E-16, 6. 16E-16/

DATA SV4/3.28E-23,1.02E-22,2.52E-22,5.29E-22,9.84E-22, $1.68 \mathrm{E}-21,2.66 \mathrm{E}-21,4.00 \mathrm{E}-21,5.75 \mathrm{E}-21,7.96 \mathrm{E}-21$, $1.07 E-20,1.40 E-20,1.79 E-20,2.24 E-20,2.76 E-20$, $3.35 \mathrm{E}-20,4.01 \mathrm{E}-20,4.75 \mathrm{E}-20,5.56 \mathrm{E}-20,6.45 \mathrm{E}-20$, $7.42 E-20,8.47 E-20,9.60 E-20,1.08 E-19,1.21 E-19$, $1.35 \mathrm{E}-19,1.49 \mathrm{E}-19,1.64 \mathrm{E}-19,1.80 \mathrm{E}-19,1.97 \mathrm{E}-19$, 2.14E-19,2.32E-19,2.51E-19,2.71E-19,2.91E-19, $3.12 E-19,3.34 E-19,3.56 E-19,3.79 E-19,4.03 E-19$, 4.27E-19,4.52E-19,4 .77E-19,5.03E-19,5.30E-19, $5.57 \mathrm{E}-19,8.54 \mathrm{E}-19,1.19 \mathrm{E}-18,1.56 \mathrm{E}-18,1.96 \mathrm{E}-18$, $2.37 \mathrm{E}-19,2.80 \mathrm{E}-18,3.24 \mathrm{E}-18,3.68 \mathrm{E}-18,4.13 \mathrm{E}-18$, 4.59E-18,5.04E-18,5.50E-19,5.96E-18,6.41E-18, $6.87 \mathrm{E}-18,7.33 \mathrm{E}-18,7.78 \mathrm{E}-18,8.23 \mathrm{E}-18,8.68 \mathrm{E}-18$, $9.12 \mathrm{E}-18,9.57 \mathrm{E}-18,1.00 \mathrm{E}-17,1.04 \mathrm{E}-17,1.09 \mathrm{E}-17$, 1.13E-17, 1.17E-17, 1.22E-17, 1.26E-17, 1.30E-17, $1.34 \mathrm{E}-17,1.39 \mathrm{E}-17,1.43 \mathrm{E}-17,1.47 \mathrm{E}-17,1.51 \mathrm{E}-17$, $1.55 \mathrm{E}-17,1.59 \mathrm{E}-17,1.63 \mathrm{E}-17,1.67 \mathrm{E}-17,1.71 \mathrm{E}-17$, $1.75 \mathrm{E}-17,1.79 \mathrm{E}-17,1.83 \mathrm{E}-17,1.87 \mathrm{E}-17,1.91 \mathrm{E}-17$, $1.95 \mathrm{E}-17,2.32 \mathrm{E}-17,2.66 \mathrm{E}-17,2.97 \mathrm{E}-17,3.25 \mathrm{E}-17$, $3.48 \mathrm{E}-17 /$

DATA SV5/0.00E-00,0.00E-00,0.00E-00,0.00E-00,0.00E-00, $0.00 \mathrm{E}-00,0.00 \mathrm{E}-00,0.00 \mathrm{E}-00,0.00 \mathrm{E}-00,0.00 \mathrm{E}-00$, $0.00 \mathrm{E}-00,0.00 \mathrm{E}-00,0.00 \mathrm{E}-00,0.00 \mathrm{E}-00,0.00 \mathrm{E}-00$, $0.00 E-00,0.00 E-00,0.00 E-00,0.00 E-00,0.008-00$, $0.00 E-00,0.00 E-00,0.00 E-00,0.00 E-00,0.00 E-00$, $0.00 \mathrm{E}-00,0.00 \mathrm{E}-00,0.00 \mathrm{E}-00,0.00 \mathrm{E}-00,0.00 \mathrm{E}-00$, $0.00 \mathrm{E}-00,0.00 \varepsilon-00,0.00 \mathrm{E}-00,0.00 \mathrm{E}-00,0.00 \mathrm{E}-00$, $0.00 E-00,0.00 E-00,0.00 E-00,0.00 E-00,0.00 E-00$, 
$0.00 E-00,0.00 E-00,0.00 E-00,0.00 E-00,0.00 E-0 ;$, 1. 16E-20,6.17E-20,1 . 12E-19,1 .62E-19,2.12E-19, $2.62 E-19,4.37 E-19,6.11 E-19,7.85 E-19,9.60 E-19$, $1.13 \mathrm{E}-18,1.47 \mathrm{E}-18,1.80 \mathrm{E}-18,2.14 \mathrm{E}-18,2.47 \mathrm{E}-18$, $2.81 \mathrm{E}-18,3.30 \mathrm{E}-18,3.80 \mathrm{E}-18,4.29 \mathrm{E}-18,4.79 \mathrm{E}-18$, $5.29 \mathrm{E}-18,5.93 \mathrm{E}-18,6.58 \mathrm{E}-18,7.22 \mathrm{E}-18,7.87 \mathrm{E}-18$, $8.52 E-18,0.29 E-18,1.01 E-17,1.09 E-17,1.16 E-17$, $1.24 \mathrm{E}-17,1.33 \mathrm{E}-17,1.42 \mathrm{E}-17,1.51 \mathrm{E}-17,1.60 \mathrm{E}-17$, $1.69 \mathrm{E}-17,1.78 \mathrm{E}-17,1.88 \mathrm{E}-17,1.98 \mathrm{E}-17,2.08 \mathrm{E}-17$, 2.18E-17,2.29E-17,2.39E-17.2.50E-17,2.61E-17, 2.71E-17, 4.01E-17,5.30E-17,6.59E-17, 7.88E-17, 9.18E-17'

DATA SV6/3. 10E-26, 1.80E-25,7.28E-25,2.31E-24,6.11E-24, ! $1.41 E-23,2.94 E-23,5.63 E-23,1.01 E-22,1.70 E-22$, $2.73 E-22,4.22 E-22,6.29 E-22,9.09 E-22,1.28 E-21$, $1.75 E-21,2.36 E-21,3.11 E-21,4.03 E-21,5.14 E-21$, $6.46 \mathrm{E}-21,8.03 \mathrm{E}-21,9.87 \mathrm{E}-21,1.20 \mathrm{E}-20,1.45 \mathrm{E}-20$, 1.73E-20,2.05E-20,2 . 41E-20,2 2 श E-20,3.26E-20, 3.76E-20,4 . 32E-20,4 . 93E-20,5.61 E-20,6.35E-20, 7. 15E-20,8.03E-20,8.98E-20,1.00E-19,1.11E-19, $1.23 \mathrm{E}-19,1.36 \mathrm{E}-19,1.50 \mathrm{E}-19,1.65 \mathrm{E}-19,1.81 \mathrm{E}-19$, $1.97 \mathrm{E}-19,4.33 \mathrm{E}-19,8.17 \mathrm{E}-19,1.39 \mathrm{E}-18,2.19 \mathrm{E}-18$, $3.26 E-18,4.61 E-18,6.28 E-16,9.27 E-18,1.06 E-17$, $1.32 \mathrm{E}-17,1.62 \mathrm{E}-17,1.95 \mathrm{E}-17,2.30 \mathrm{E}-17,2.68 \mathrm{E}-17$, 3. $09 \mathrm{E}-17,3.52 \mathrm{E}-17,3.96 \mathrm{E}-17,4.42 \mathrm{E}-17,4.89 \mathrm{E}-17$, $5.37 \mathrm{E}-17,5.87 \mathrm{E}-17,6.36 \mathrm{E}-17,6.87 \mathrm{E}-17,7.37 \mathrm{E}-17$, $7.88 \mathrm{E}-17,8.38 \mathrm{E}-17,8.88 \mathrm{E}-17,9.38 \mathrm{E}-17,9.87 \mathrm{E}-17$, $1.04 \mathrm{E}-16,1.08 \mathrm{E}-16,1.13 \mathrm{E}-16,1.18 \mathrm{E}-16,1.22 \mathrm{E}-16$, $1.27 E-16,1.31 E-16,1.36 E-16,1.40 E-16,1.44 E-16$, $1.48 \mathrm{E}-16,1.52 \mathrm{E}-16,1.56 \mathrm{E}-16,1.60 \mathrm{E}-16,1.63 \mathrm{E}-16$, $1.67 \mathrm{E}-16,1.97 \mathrm{E}-16,2.19 \mathrm{E}-16,2$. 34E-16, 2.45E-16, $2.52 \mathrm{E}-16 /$

SVEUSE $=0$.

$T=T I / 1000$.

IF(T.LT.1. AAND. IRATE.EQ.3) GO TO 20

IF(T.LT.1. .OR. T.GT. 200.) RETURN

DTX $=.2$

IPT $=-4$

IF(T.LT.10.) GOTO 10

DTX $=2$.

$I P T=41$

IF(T.LT.100.) GOTO 10

DTX $=20$.

IPT $=86$

$10 I X=I F I X(T / D T X)+I P T$

$T X=D T X "(I X-I P T)$

SVFUSE $=S V(I X$, IRATE $)+(S V(I X+1$, IFATE $)-S V(I X$, IRATE $)) *(I-I X) / D T X$ RETURN

$S V L=((-1.110333 * T L+12.2(350) * T L-49.01817) * T L+86.86500$

IF (SVL.: $\therefore .37$.$) SVFUSE =10 . \cdots(-$ SVL)

C THIS FIT IS GOOD TO SVL $=50$. BUT DEC MACHINES CAN'T HANDLE 1.E-50 RETURN

END 
Appendix E Sample Cases

Following are two test runs. The first example is oitained with the impurity density, NZ, flagged as uriknosn. In the second example $\mathrm{NZ}$ is flagged as a preferred value. A comparison of the two shows that $N Z$ stayed closer to its initial value when marked preferred.

The file ETFOD.OUT contains the input data from ETFOD.DAT followed by three parameters fixed in the code. The initial residuals of the physics equations are listed next. Following those is the trace of the iterative process in E04FAF as output by MONIT. Upon exit from E04FAF, a statement is written in this file indicating whether a solution has or has not been found. Following this are some plasma parameters, the final residuals of all the equartons, the accuracy of the physics equations, the normalized solution vector, and the final values of the input variables.

In these examples some of the intermediate princout from MONIT has been left out due to the length of the listing.

The file SPECS.ETF contains the final design specifications. 
File ETFOD.OUT (Example 1)

$10.12008+02$ (REV)

$0.1500 \mathrm{E}+01$

$50.1700 \mathrm{E}+21$

5 0.17008+2

$10.30008+01$

$0.70008-01$

$9.50104 \times 01$

$120.10428+11$

$130.33008+08$

$0.13408+01$

$150.11798+01$

$160.16005+01$

$170.00005+00$

$180.10008-23$

190.100

$200.10008+01$

$210.50008+07$

$\begin{array}{lll}\text { F II } & \text { (REV) } \\ \text { P } & \text { IE } & \text { (REV) } \\ \text { IEFF } & \end{array}$

2EFF

MI $(M \times-3)$

n2 (MT-3)

- (SAFETY TACTOR)

BT (TESLA)

TAUE (SEC)

PALFV (W/Me'3)

Prus (U)

HPA (U/HOC)

A

(A)

An

PIom (W)

(W)

CI

(MPS)

meste: Farixed value

PapREFEnAE VALUE

$x \mathrm{xTH}=1.0000005-01$

$\operatorname{stchex}: 5.0000008-01$

SUM or sovines $\quad 0.0000$

RESI DUALS:

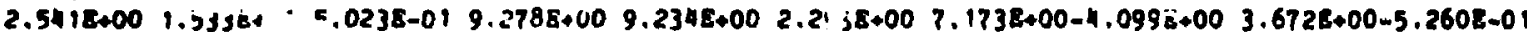

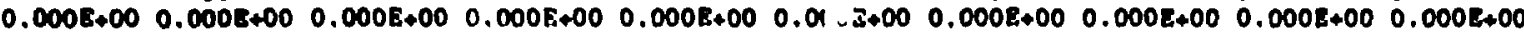

$0,0008+00 \quad 0.0008+00$

SUM OF SQUARES : 5003E+03 AFTER O ITERATIONS

SIm - F (IF

CUnnest solutron:

$\begin{array}{llllllllll}1.000 \varepsilon+00 & 1.000 \varepsilon+00 & 1.000 \varepsilon+00 & 1.0008+00 & 1.0008+00 & 1.0008+00 & 1.0008+00 & 1.0008+00 & 1.0008+00 & 1.0008+00\end{array}$

$1.0008+001.0008+00$ 


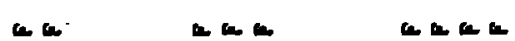

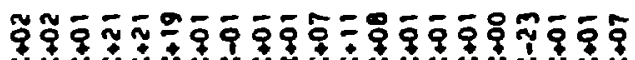

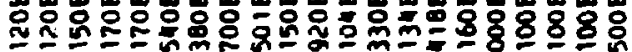

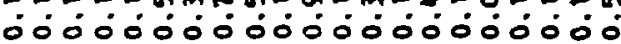

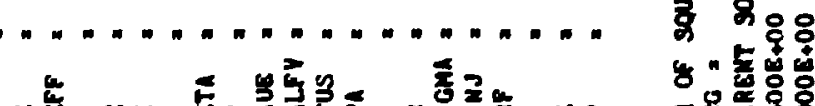

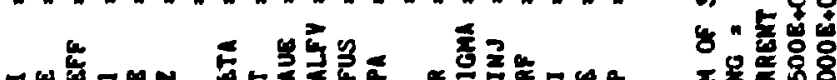

ab a late

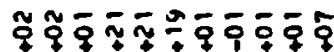

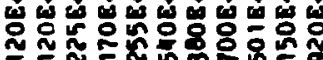

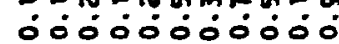

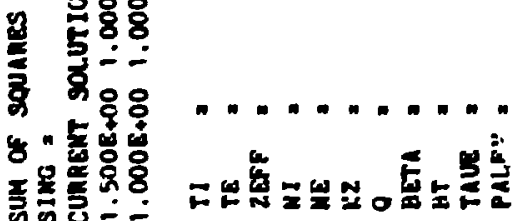




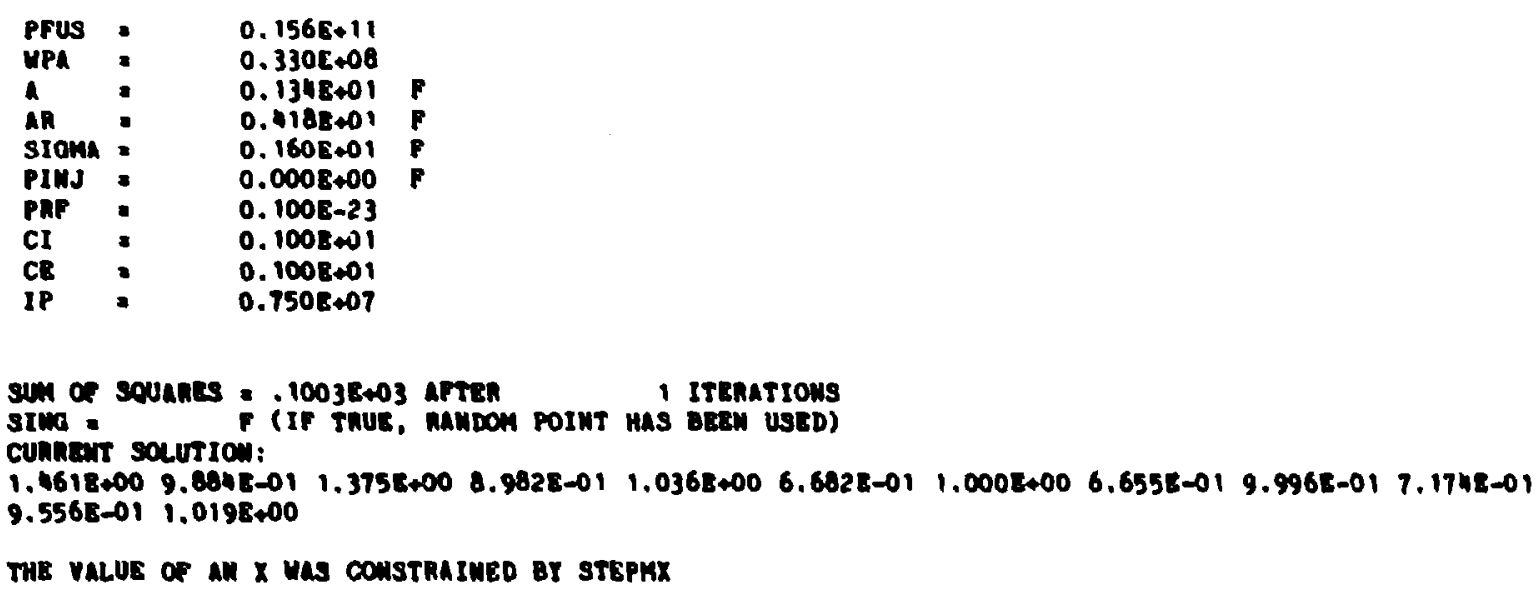

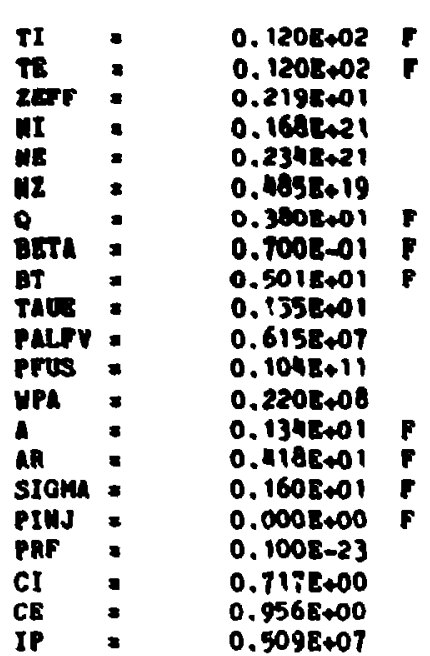


SUM OF SQUARES : $19508+02$ AFTER
SIMG = 2 ITERATIONS
CURREET SOLUTION:

$1.3968+00 \quad 9.823 E-01 \quad 1.245 E+00 \quad 7.564 E-01 \quad 1.027 E+00 \quad 3.3648-01 \quad 5.000 E-01 \quad 3.309 E-01 \quad 9.833 E-01 \quad 6.733 E-01$ $8.291 \mathrm{k}-011.038 \mathrm{E}+00$

THE VALUE OF AN $X$ WAS CONSTRAIMED BY STEPHX

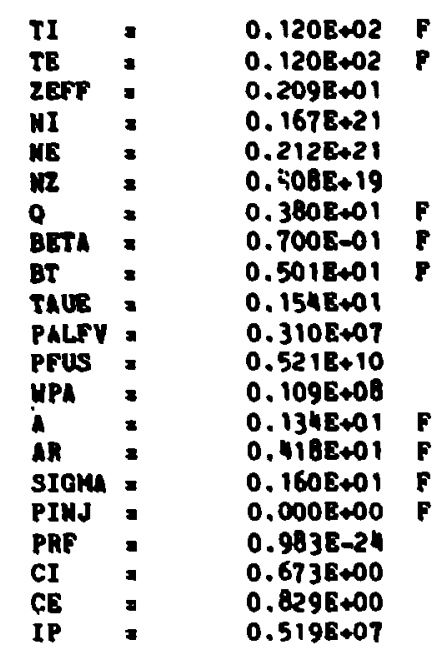

SUH OP SQUARES : 7881E-02 AFTER
SING
TIF TRUE, RANDON POINT HAS BEEN USED)

ClisRent SOLUTION:

1.268E.00 9.6808-01 1.133E+00 5.709E-01 1.006E+00 7.501E-02 1.058E-01 6.695E-02 9.302E-01 5.103E-01 $7.8238-01,1.0538+00$
TI
$0.1208+02$ 


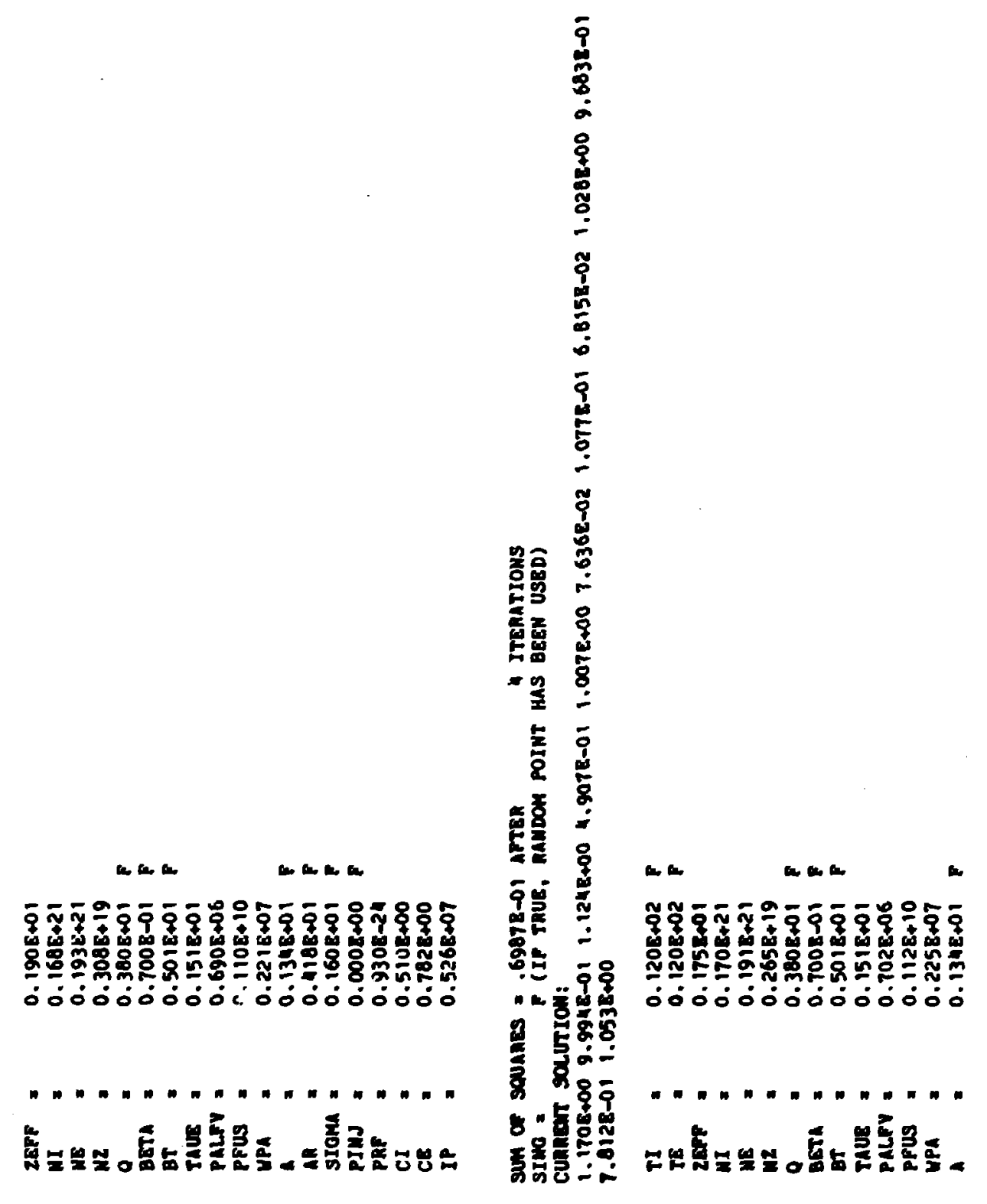




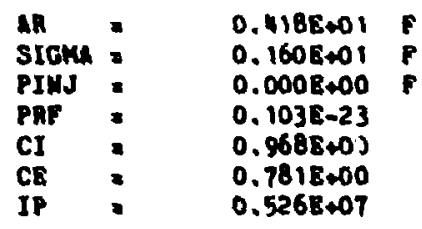

SUN OF SOUARES = .1224E-01 AFTER 5 ITERATIONS SING = P (IF TRUE, RANDON POINT HAS BEEN USED)

CUnAarr soutrion:

179E+00 9.9948-01 1.1248+00 \$.9118-01 9.883E-01 7.662E-02 1.060E-01 6.8398-02 8.864E-01 6.6758-01 $1.1468-01 \quad 1.0538+00$

\begin{tabular}{|c|c|c|}
\hline 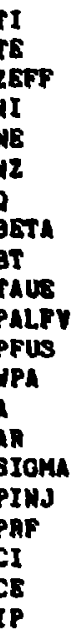 & $=$ & $\begin{array}{l}0.1208+02 \\
0.1208+02 \\
0.1778+01 \\
0.1708+21 \\
0.1918+21 \\
0.2658+19 \\
0.3808+01 \\
0.7008+01 \\
0.5018+01 \\
0.148 E+01 \\
0.7058+06 \\
0.1138+10 \\
0.2268+07 \\
0.1348+01 \\
0.4188+01 \\
0.1608+01 \\
0.0008+00 \\
0.0868-24 \\
0.6678+00 \\
0.715 E+00 \\
0.5268+07\end{array}$ \\
\hline
\end{tabular}

SUH OP SOUARES : 10768+02 APTER ITEATIONS

SIWG = I (IF TAUE, RANDON POINT HAS BEEN USED) 


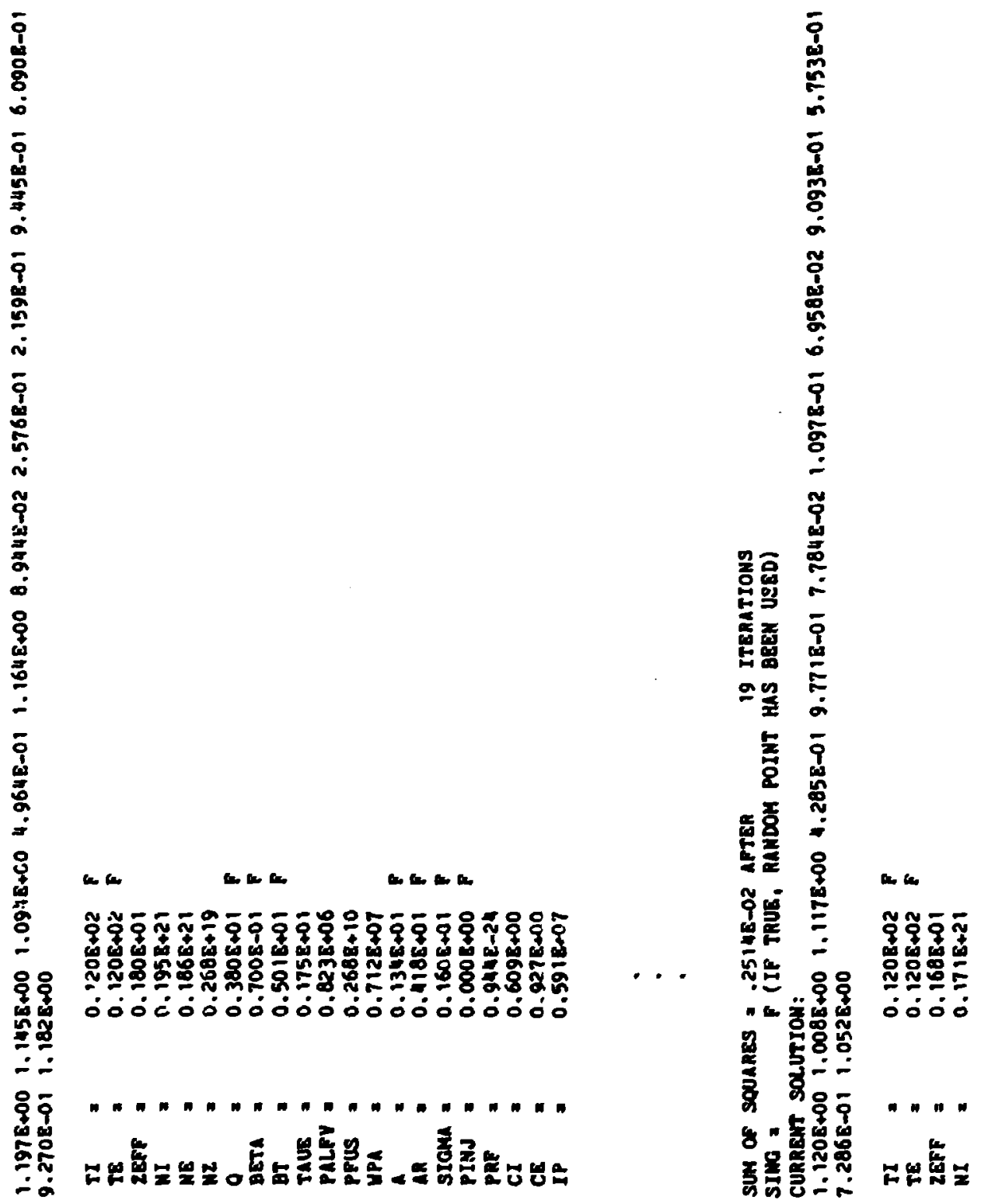




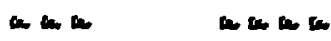

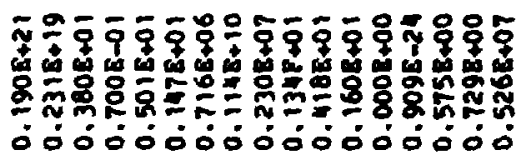

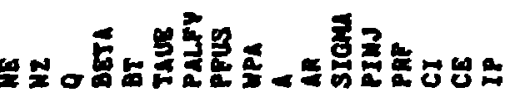

\$

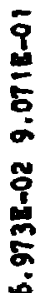

言查

할

E

㩆焉

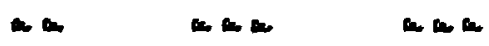

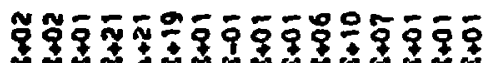

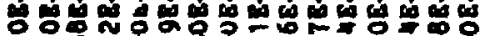

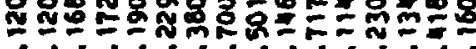

"

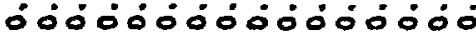

政

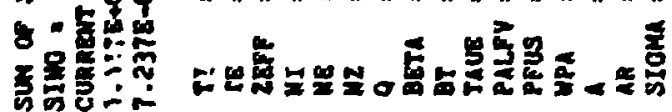

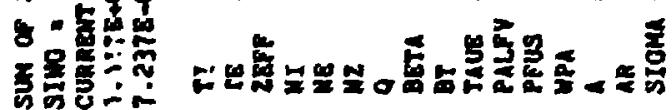




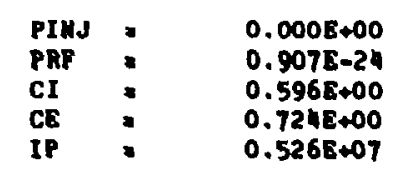

SUM OF SQUARES I 2105E-02 AFTER IIF TRUE, MANDOH POINT HAS BEEM USED)
SIMG =

CURReirt souUtion:

$1.1238+00 \quad 1.0088+00 \quad 1.117 \varepsilon+00$ 4. 3068-01 9.7698-01 7.7798-02 1.097E-01 6.9478-02 9.1068-01 5.7318-01 $7.2708-011.0528+00$

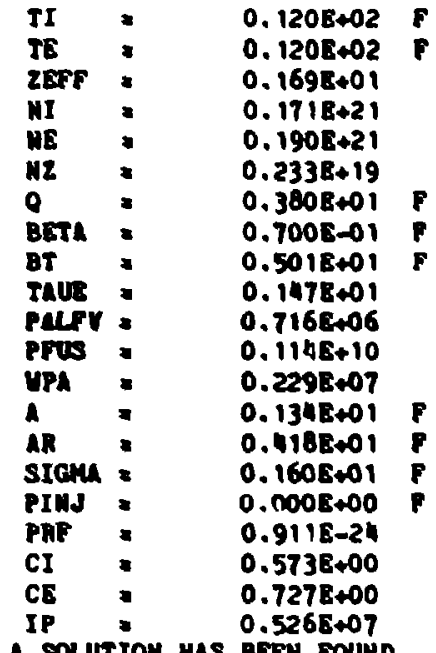

I SOLUTION HAS BEEN FOUND.

TOTAL ALPHA POUER

INTERHAL INDUCTANCE

IHDOCTIVE VOLT-SECONDS

PLAST VOLTAGE

DEUTERIUH DIFFUSION LOSS RATE

$0.2272808+03$

$0.5277598-05$

0.599751 E-0

$0.4632762-0$

$0.1957008+23$
MajOR RADIUS

EXTERHAL INDUCTANCE

PLAsha Resistamce

FRACTION OF FUBL BURNED
$0.5599868+01$
$0.8968468-05$
$0.8803468-08$
$0.4053518+21$
$0.2136208-01$ 
SUM OF SOUARES $=.2105 E-02$ FTOL $=-1,0$ IFAIL $=0$

RESIDUALS:

1.24108-03 6.6227E-03 5.6278E-04 6.92 17E-05-6.4212E-04 1.4226E-03 1. 37758-03-3.0541E-04 7.3326E-04-1, 15008-03 $4.93558-03$ 3.22 10E-04 M.69448-03-5.6937E-03-2.31348-04-9.222 1E-03-8.9035E-03-3.7221E-02-3.5760E-03-1.70768-02 $-1.09218-025.24878-04$

THIS SOUTION DOES SATISFY PHYSICS EOUATIONS WITHJN 1.08

\begin{tabular}{|c|c|c|c|c|}
\hline $\begin{array}{l}\text { EQUATION } \\
\text { EQUATION } \\
\text { EQUATION } \\
\text { EQUATION } \\
\text { EQUATION } \\
\text { EQWATION } \\
\text { EQUATION } \\
\text { EQUATION } \\
\text { EQUATION } \\
\text { BOUTION }\end{array}$ & $\begin{array}{l}1 \text { is } \\
2 \text { is } \\
3 \text { is } \\
4 \text { is } \\
5 \text { is }\end{array}$ & $\begin{array}{l}\text { BALAMCED } \\
\text { BALANCED } \\
\text { BALANCED } \\
\text { BLLANCED } \\
\text { BALANCED } \\
\text { BLLANCED } \\
\text { BLLANCED } \\
\text { BLLANCED } \\
\text { BALANCED }\end{array}$ & $\begin{array}{l}\text { HITHIN } \\
\text { UITHIN } \\
\text { YITHIN } \\
\text { HITHIN } \\
\text { UITHIN } \\
\text { UITHIN } \\
\text { UITHIN } \\
\text { UITHIN } \\
\text { WITHIN } \\
\text { UITHIN }\end{array}$ & $\begin{array}{r}0.018 \\
0.078 \\
0.018 \\
0.008 \\
-0.018 \\
0.018 \\
0.018 \\
-0.008 \\
0.018 \\
-0.018\end{array}$ \\
\hline
\end{tabular}

MOMULIZED X:

$1.1234 E+00 \quad 1.0081 E+00 \quad 1.1174 E+00 \quad 4.3063 E-01 \quad 9.76878-01 \quad 7.7793 E-02 \quad 1.09658-01 \quad 6.94678-02 \quad 9.10608-01 \quad 5.7310 E-01$ $1.2698 \mathrm{E}-01 \quad 1.0525 \mathrm{E}+00$

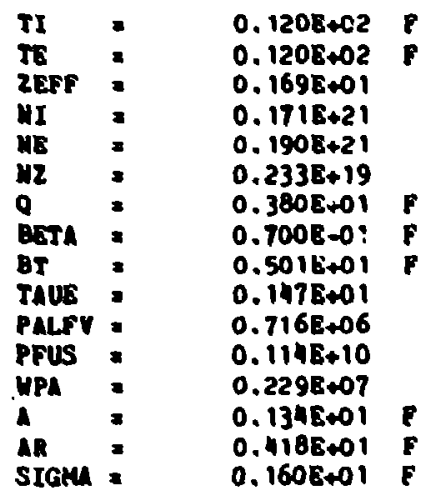




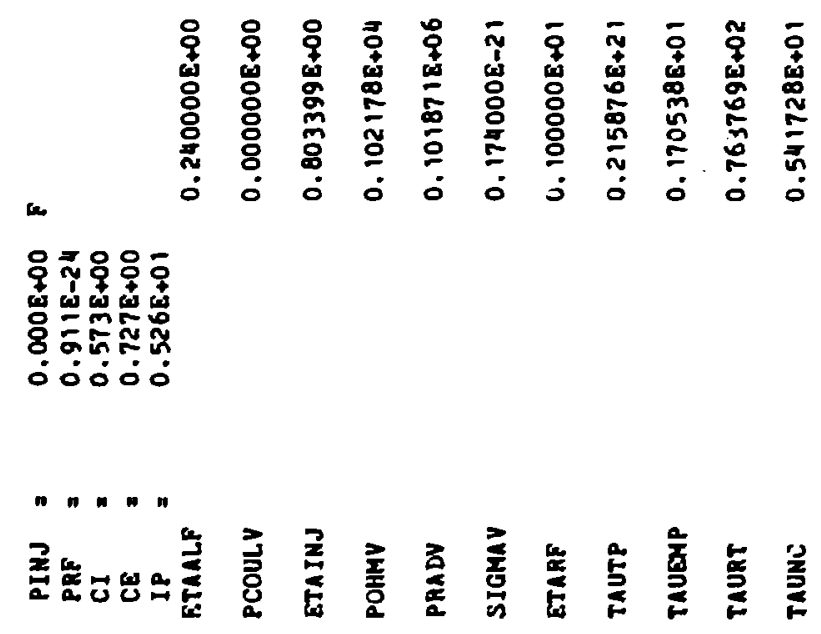


File SPECS.ETF (Example 1)

$\begin{array}{llr}\text { PLASMA RADIUS (M) } & = & 1.340 \\ \text { ASPECT RATIO } & = & 4.179 \\ \text { ELONGATION } & = & 1.600 \\ \text { BETA } & = & 0.070 \\ \text { FIELD ON AXIS(T) } & = & 5.010 \\ \text { ION TEMP (KEV) } & = & 12.000 \\ \text { ELE TEMP (REV) } & = & 12.000 \\ \text { RIPPLE ( }) & = & 1.550\end{array}$

$\begin{array}{llr}Q & = & 3.800 \\ \text { IMPURITY Z } & 8.000 \\ \text { ZEFE } & = & 1.685 \\ \text { GAMMA (PES LOSS) } & =0.100 \\ \text { PLASMA INT IND (LI) } & =1.500 \\ \text { SCRAPE CFF LAYER (M) } & =0.25 n \\ \text { IP FACTOR } & =0.800 \\ \text { BURN PRESS (TORR) } & =.100 C E-05 \\ \text { QUENCH PRESS (TORR) } & =.1000 E-03\end{array}$

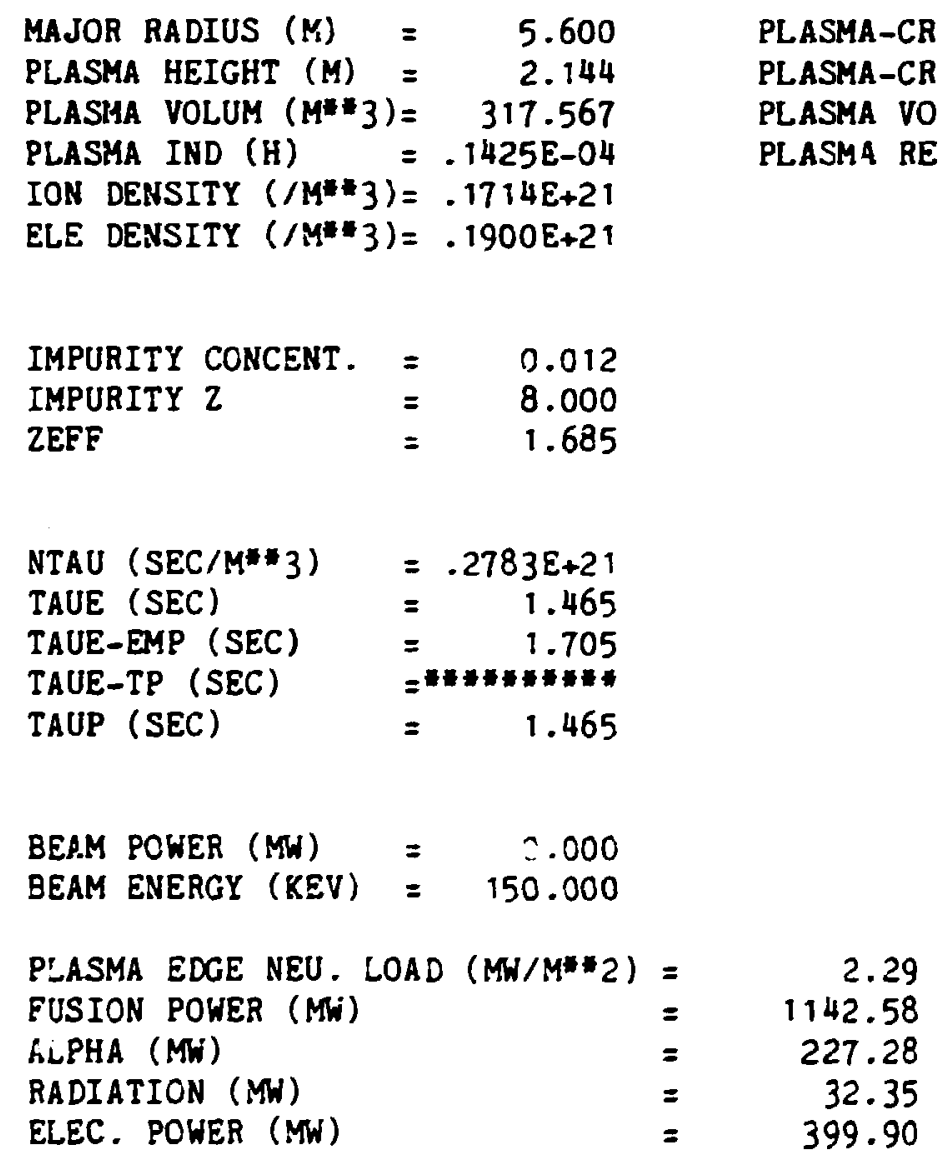


File ETFOD.OUT (Example 2)

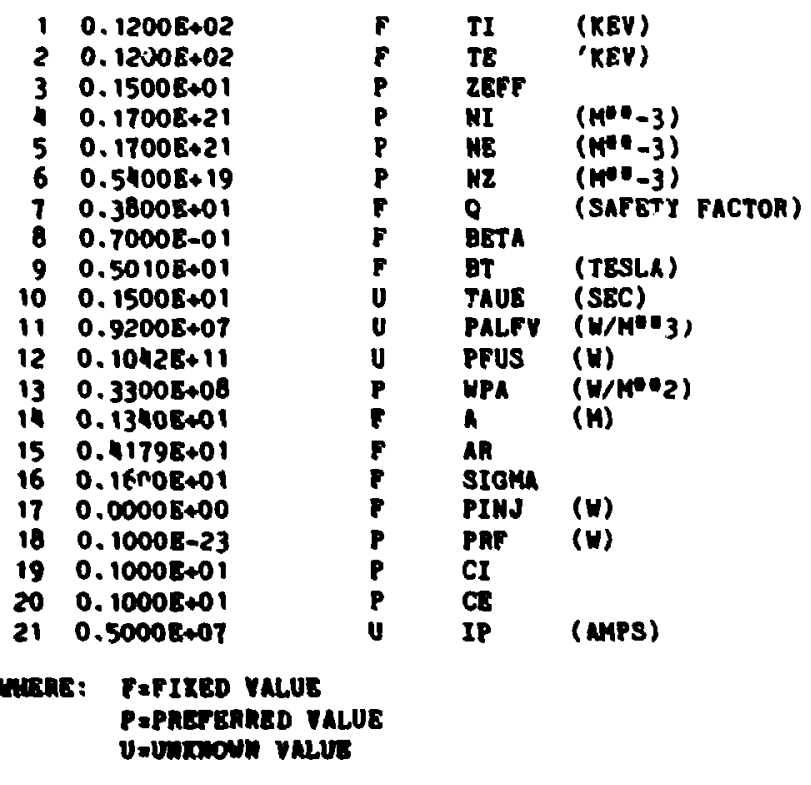

\section{$x$ TTOL $=1,0000008-01$}

STEPAX $=5.0000008-01$

FTOL = 1.0000008-06

\section{SUM OF SOUARES = 0.0000}

\section{MESIDUALS:}

$2.541 E-301.5338+01 \quad 5.0238-01 \quad 9.278 E+00 \quad 9.234 E+002.2938+00 \quad 7.1738+00-4.0998+00 \quad 3.6728+00-5.260 E-01$

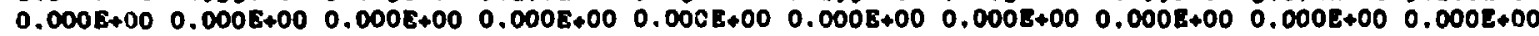
$0.0008+00 \quad 0.0008+00$

SUH OF SOUARES = $30038+03$ AFTER
SINO $=$ O ITERATIONS

CURRENT SOLUTION:

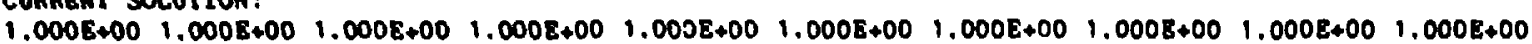
$1.0008+001,0008+00$ 


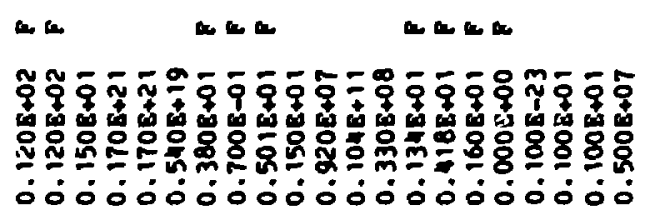

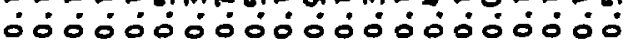

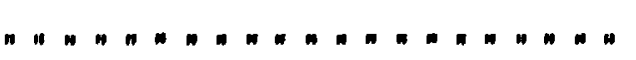

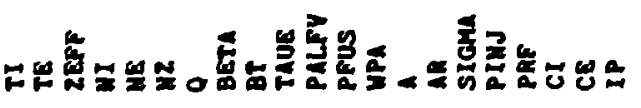

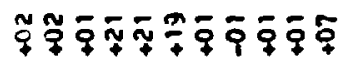

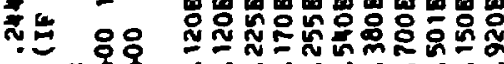
" 


$$
\begin{aligned}
& \text { PFus = } 0.1508+11 \\
& \text { UPA }=0.330 E+08 \\
& \text { a }=0.1348+01 \\
& \text { An : } 0.4188+01 \\
& \text { stome }=0.160 E+01 \\
& \text { PINJ = } 0.000 \mathrm{E}+00 \mathrm{~F} \\
& \text { PRF }=0.100 E-2 \\
& \mathrm{CI}=0.100 \mathrm{E}+01 \\
& \text { CE } .0 .100 \mathrm{E}+01 \\
& \text { IP } \therefore \quad 0.750 E+07
\end{aligned}
$$

SUH OF SMARES = .1011E+03 AFTER i ITERATIONS

SING : F (IF TRUE, MANDOH. POINT HAS LEEN USED)

CURABUT SOLUTION:

$1.4068+009.0518-01 \quad 1.378 E+00 \quad 9.21>8-01 \quad 1.0468+00 \quad 6.6118-01 \quad 1.0008+00 \quad 6.6558-01 \quad 1.0008+00 \quad 7.2378-01$ $9.8628-01,1.0195+00$

THE VALUE OF AN $x$ WAS CONSTRAINED GY STEPHX

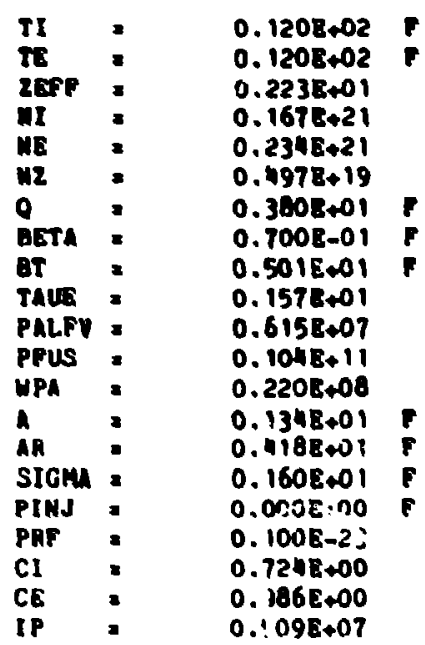


SUM OF SQUARES : 1984E+02 AFTER

CURREIT SOLUTION.

1.457E-00 9.729E-01 1.253E+00 8.231E-01 1.050E+nO 3.363E-01 5.000E-01 3.310E-01 9.835E-01 6.731E-01

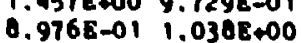

THE VALUE OF AN $X$ WAS COWSTRAINED gY STEPHX

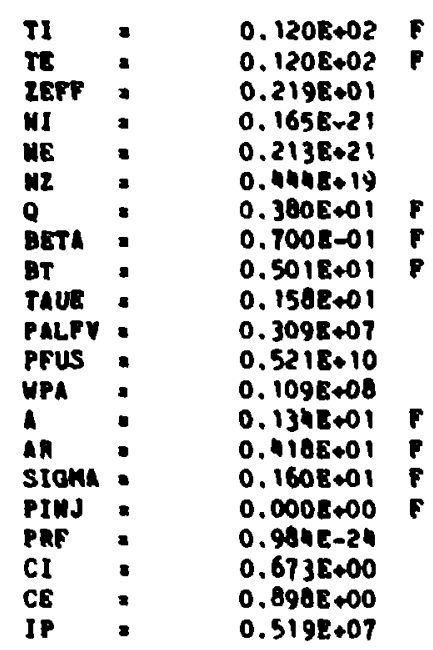

SUM OF sOWARES : j025E-02 AFTER
SIMO

CUAnEart solution:

1.409E+00 9.669E-01 1.149E+00 7.183E-0 1 1.057E+00 7.155E-02 1.0098-01 6.306E-02 9.554E-01 5.282E-01 $0.1408-011.0538400$

TI: $0.1208+02 \mathrm{~F}$ 


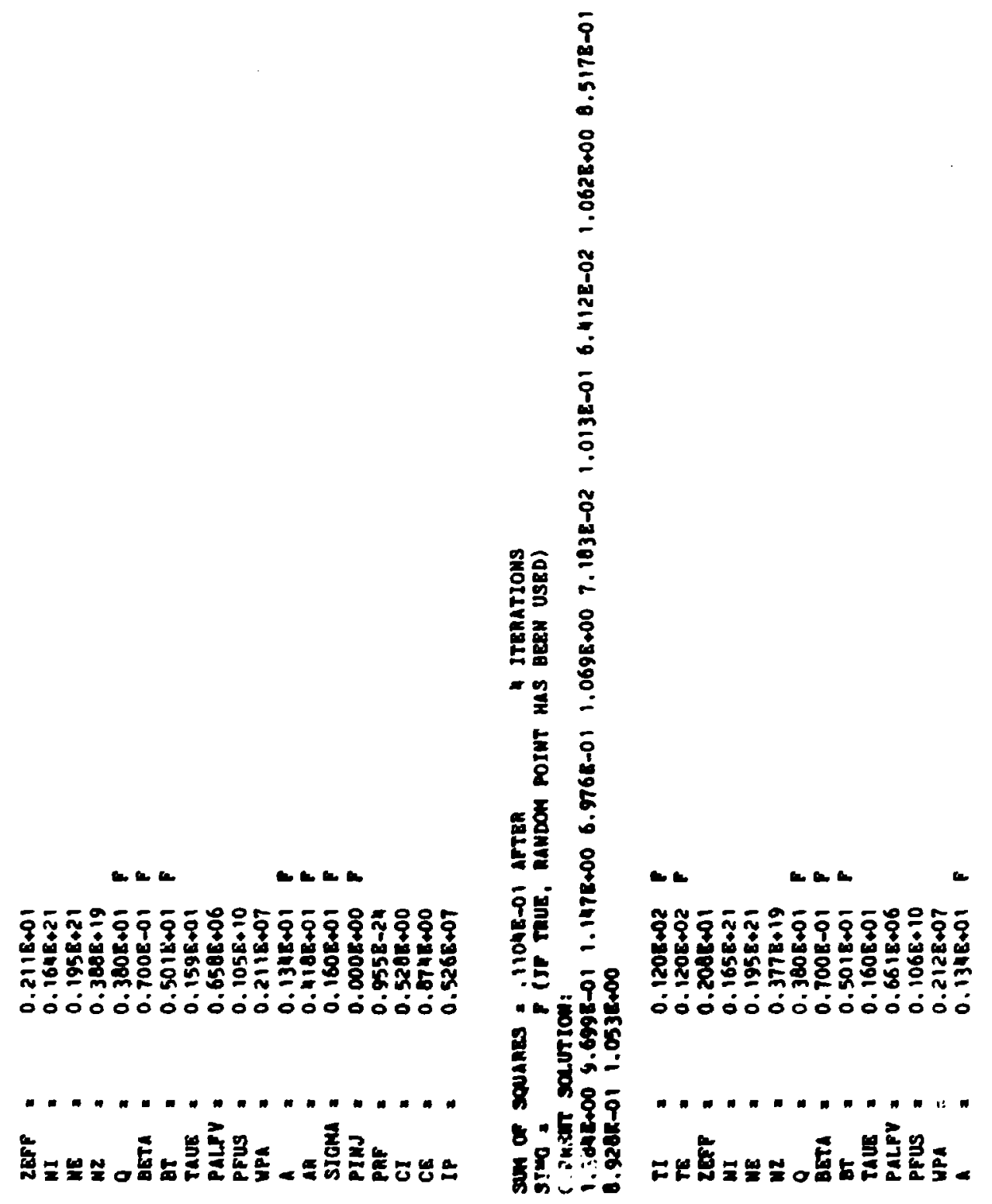




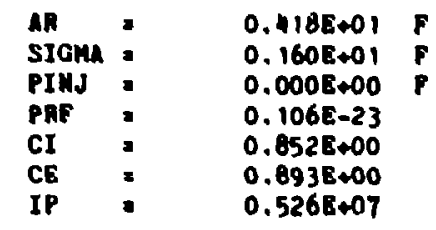

SUM Of SQUARES : .1167E-OI AFTEA 5 ITEATIONS

SImo. (IF TRUE, RAHDON POINT HAS OFEN USED)

CURREAT SOLUTION:

$1.358 E+00$ 9.734E-01 1.144E+00 6.726E-01 1.041E+00 7.278E-02 1.0268-01 6.496E-02 8.662E-01 6.408E-01

B. $1708-011.0538=00$

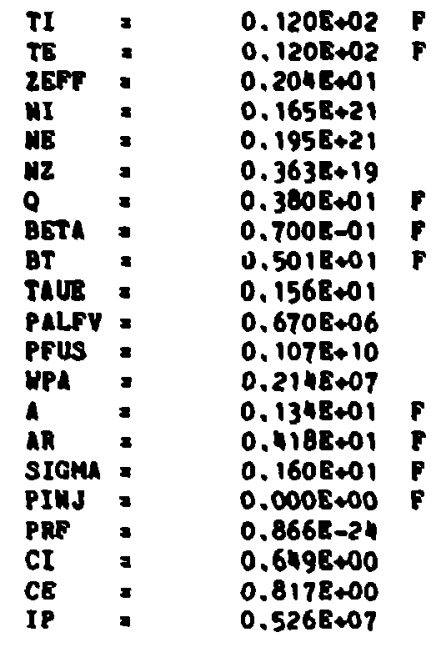

SUM OF SQUARES $.2378 E+02$ AFTER 6 ITERATIONS

SIMO

CURAENT SOLUTION: 


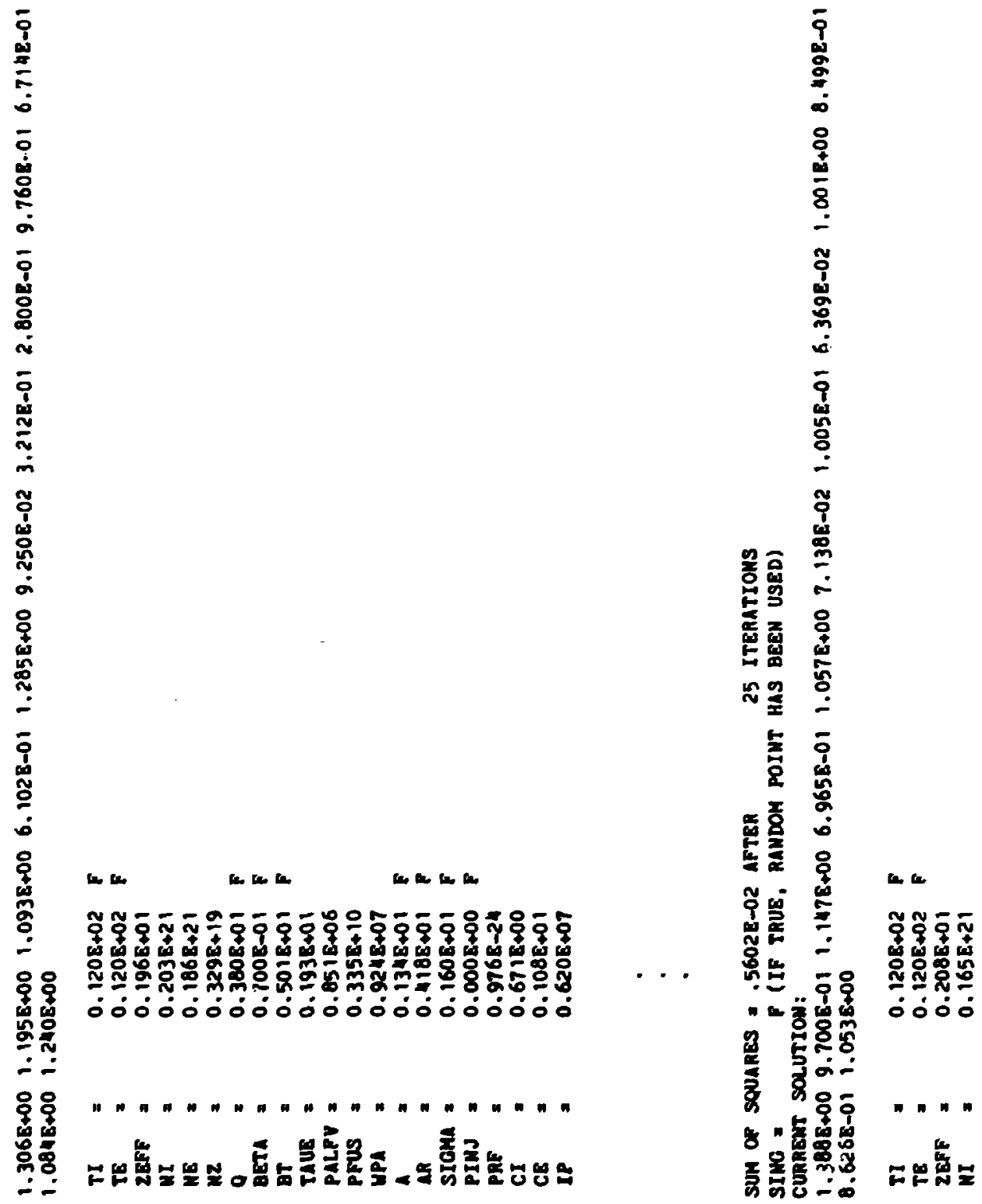




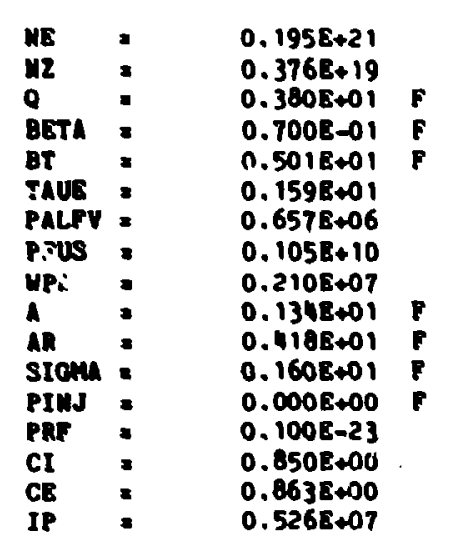

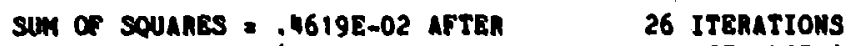

SIMO = (IF TRUE, RAMDOH POINT HAS BEEN USED)

CURnEar solution:

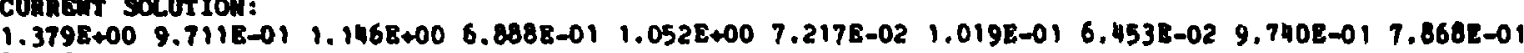

$8.5968-01,1.0536400$ 


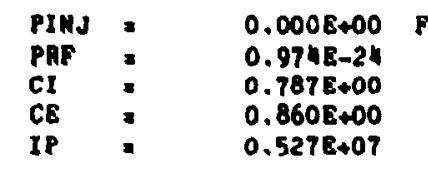

SUM OF SQUARES :2525E-02 AFTER POT ITERATIONS
SIMG

CURREAT SOLUTYON:

$1.3898+00 \quad 9.6948-01 \quad 1.1488+00 \quad 6.993 E-01 \quad 1.0568+00 \quad 7.1988-02 \quad 1.014 E-01 \quad 6.4108-029.5508-01 \quad 6.162 E-01$ $0.6678-01 \quad 1.0528+00$

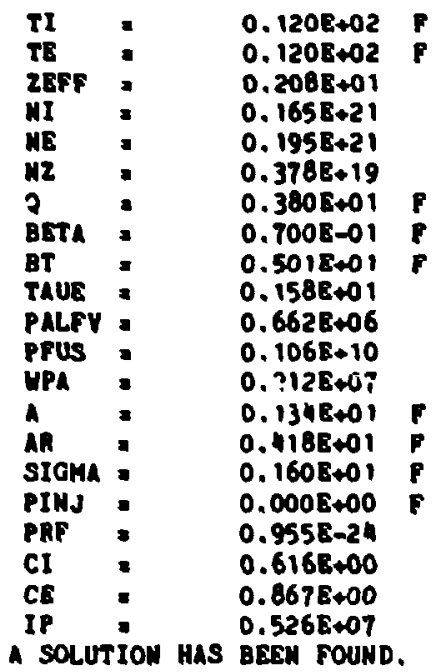

TOTAL ALPHA POUER

IHTERMAL INDUCTANCE

INDUCTIVE VOLT-SECONDS

PLASMA VOLTAGE

$0.527759 \mathrm{E}-05$

$0.5998208-0$

DEUTERIUH DIFFUSION LOSS RATE

$0.165004 \mathrm{E}+23$

MAJOR RADIUS

EXTERHAL IHDUCTANCE

PLASMA hesistance

PRACTION OF PUEL BURAED
$0.5599868+01$
$0.8968468-05$
$0.109192 E-07$
$0.3742008+21$
$0.2217538-01$ 
SUA OF SOUARES = .2334E-02 FTOL =-1.0 IFAIL = 0

RESI DUALS:

$-1.0218 E-03 \quad 1.0623 E-03-2.59448-04-7.4855 E-04-1.64718-03 \quad 1.96128-03 \quad 1.38498-03 \quad 1.2088 \varepsilon-03 \quad 6.24658-04 \quad 5.56258-05$ 1.5671E-02-1.2298E-03 5.9087E-03-1, 1925E-02 5.7077E-04-9.2819E-03-8.9862E-03-3.74318-02-2 .2454E-03-1.7202E-02 $-5.2006 \mathrm{E}-03 \quad 5.2607 \mathrm{E}-0 \mathrm{H}$

THIS SOLTION DORS SATISFY PHYSICS EQUATIONS WITHIN 1.08

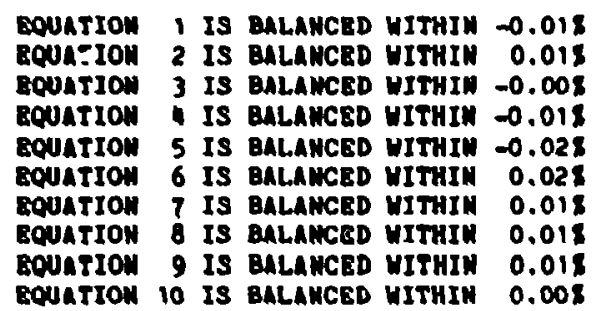

EQUaTION 10 is BALAMCED UITHIM 0.008

MOPMALI2ED $X$

1.3918E+00 9.6925E-01 1,1477E+00 7,0188E-01 1,05718+00 7,1814E-02 1,01388-01 6.4218E-02 9.4386E-01 5,69958-01

$8.69998-011.0526 E+00$

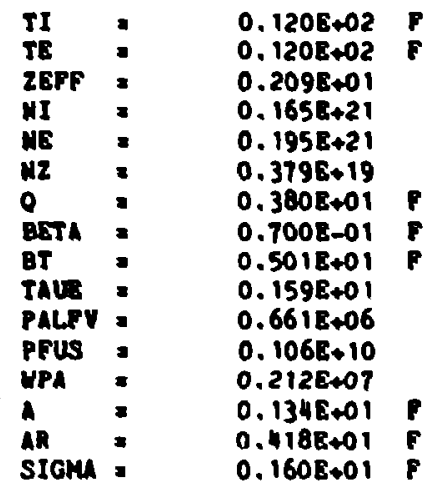



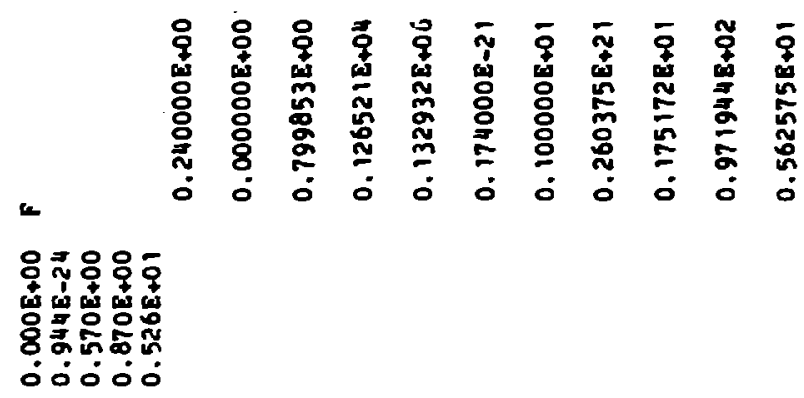

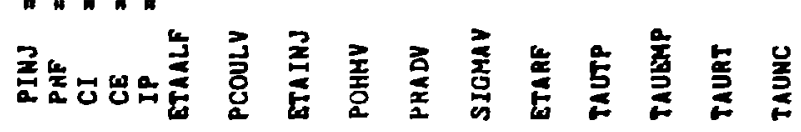


File SPECS.ETF (Example 2)

$\begin{array}{llr}\text { PLASMA RADIUS (M) } & & 1.340 \\ \text { ASPECT RATIO } & = & 4.179 \\ \text { ELONGATION } & = & 1.600 \\ \text { BETA } & = & 0.070 \\ \text { FIELD ON AXIS(T) } & = & 5.010 \\ \text { ION TEMP (KEV) } & = & 12.000 \\ \text { ELE TEMP (KEV) } & = & 12.000 \\ \text { RIPPLE (q) } & = & 1.550\end{array}$

Q $=3.200$

IMPURITY Z $2=8.000$

ZEFE $\quad=2.088$

GAMMA (KES LOSS) $=0.100$

PLASMA IN ${ }_{i}^{-1}$ IND (LI) $=1.500$

SCRAPE OFE LAYER (M) $=0.250$

IP FACTOR $=0.800$

BURN PRESS (TORR) $=.1000 E-05$

QUENCH PRESS (TORR) $=.1000 E-03$

$\begin{array}{lrlr}\text { MAJOR RADIUS }(M)= & 5.600 & & \text { PLASMA-CRNT-HB (MA) }=5.263 \\ \text { PLASMA HEIGHT }(M)= & 2.144 & \text { PLASMA-CRNT-LB (MA) }= & 4.210 \\ \text { PLASMA VOLUM (M* 3) }= & 317.567 & \text { PLASMA VOLT-BURN (V) }= & 0.057 \\ \text { PLASHA IND (H) }=.1425 E-04 & \text { PLASMA RES-BURN (OHM) }=.1092 E-07\end{array}$

ION DENSITY $(/ M * 3)=.1648 E+21$

ELE DENSITY $(/ M * 3)=.1951 E+21$

$\begin{array}{llr}\text { IMPURITY CONCENT. } & = & 0.019 \\ \text { IMPURITY } 2 & = & 8.000 \\ \text { ZEFF } & & 2.088 \\ & & \\ \text { NTAU (SEC/M*3) } & = & .3094 E+21 \\ \text { TAUE (SEC) } & = & 1.586 \\ \text { TAUE-EMP (SEC) } & = & 1.752 \\ \text { TAUE-TP (SEC) } & = & 1.586 \\ \text { TAUP (SEC) } & = & \\ & & \\ \text { BEAM POWER (MN) } & = & 0.000 \\ \text { BEAM ENERGY (KEV) } & = & 150.000\end{array}$

PLASMA EDGE NEU. LOAD (MW/M*:2) = FUSION POHER (MW)

ALPHA (MW)

RADIATION (MH)

ELEC. POWER (MW)

$\begin{array}{rr}= & 2.12 \\ = & 1056.33 \\ = & 209.81 \\ = & 42.21 \\ = & 369.72\end{array}$

